\title{
The edge regions in tergites of the desert isopod Hemilepistus reaumuri: the transition from hard cuticle to flexible arthrodial membrane
}

\author{
Franziska Ernst ${ }^{1} \cdot$ Helge-Otto Fabritius ${ }^{2,3} \cdot$ Erika Griesshaber $^{4} \cdot$ Wolfgang W. Schmahl $^{4} \cdot$ Andreas Ziegler $^{1}$ (]
}

Received: 26 June 2020 / Accepted: 29 August 2020

(c) The Author(s) 2020

\begin{abstract}
The arthrodial membrane is a thin and flexible type of cuticle that inserts at the edge regions of neighbouring rigid skeletal elements creating a flexible connection. In the present study, we analyzed the structure, mineral composition, calcite organization and local stiffness and hardness of edge regions that form transitions to the arthrodial membranes in the tergites of the desert isopod Hemilepistus reaumuri. For the transitions to the arthrodial membrane, the results show an increase in the thickness of the epicuticle at cost of the distal exocuticle and a calcite layer, an increase in the ratio of phosphorus to calcium and a decrease in the local mechanical properties. The posterior edge region contains an unusually large stack of unidirectionally oriented parallel fibrils projecting to the lateral sides. At the edge, it turns down into a long ventral cuticle overlapping an anterior part of the neighbouring tergite. It forms a thin arched gap between the tergites that can help reducing water loss through the arthrodial membrane and protects the arthrodial membrane upon predation. A thick ventral ridge near the transition to the arthrodial membrane carrying bristles can prevent sand grains from access to the arthrodial membrane. From the dorsal cuticle to the transition to the arthrodial membrane, calcite units become larger and single crystalline turning their c-axes orientation perpendicular to the sagittal section plane. Comparison with edge regions of the beach isopod Tylos europaeus reveal common characteristics of the edge region, but also specific adaptations to the desert habitat of $H$. reaumuri.
\end{abstract}

Keywords Biological-material functionalization $\cdot$ Crustacea $\cdot$ Cuticle $\cdot$ Amorphous calcium phosphate $\cdot$ Calcite co-orientation strength

Electronic supplementary material The online version of this article (https://doi.org/10.1007/s00339-020-03961-0) contains supplementary material, which is available to authorized users.

Andreas Ziegler

andreas.ziegler@uni-ulm.de

1 Central Facility for Electron Microscopy, University of Ulm, Albert-Einstein-Allee 11, 89069 Ulm, Germany

2 Bionics and Materials Development, Hamm-Lippstadt University of Applied Sciences, Marker Allee 76-78, 59063 Hamm, Germany

3 Department of Microstructure Physics and Alloy Design, Max-Planck-Institut für Eisenforschung GmbH, Max-Planck-Str. 1, 40237 Düsseldorf, Germany

4 Department of Earth and Environmental Sciences, LMU, Theresienstr. 41, 80333 Munich, Germany

\section{Introduction}

The cuticle of Crustacea has a complex hierarchical organization which has attracted extensive interest during the last decade [1]. Its basic functions are that of an exoskeleton, providing support for muscles and that of a protective integument with sensory functions. The exoskeleton of Crustacea [2-5] covers the whole animal. Therefore, the cuticle is segmented into skeletal elements, most of them being tough and rigid to provide protection. To allow for relative movements, the skeletal elements are connected to each other by regions of rather thin and flexible cuticle, called arthrodial membranes. Depending on the specific tasks of cuticle regions, their physical and chemical characteristics vary considerably. These variations have evolved in a way in that the dimensions and structure of organic layers, the composition and distribution of various mineral phases and even the crystallographic characteristics of the distal calcite layer have been adapted to the specific function of a 
skeletal element, and the habitat and behaviour of the animal [2-12]. Such adaptations related to function, ecology and behaviour can affect most of the seven levels of structural hierarchy found in the crustacean cuticle (for detailed review see [1]). At the molecular level, the organic phase of the cuticle consists of $\mathrm{N}$-acetyl glucosamine monomers forming $\alpha$-chitin. The chitin is organized into $300 \mathrm{~nm}$ long and $2.5 \mathrm{~nm}$ thick crystalline nanofibrils consisting of 18-25 antiparallel polymer chains [13]. These chitin fibrils are coated with proteins and can be bundled to form thicker chitin-protein fibres. Either the fibrils or the fibres arrange in parallel and assemble to planes. The cuticle grows through continuous formation of additional fibre planes by the epithelial cells. The angle of fibre orientation can be gradually offset in superimposed planes, resulting in a helical organization sometimes termed a twisted plywood structure [14]. In the full cuticle, the stacked fibre planes form layers with distinct structural and chemical properties. The two main distinct layers are the exo- and the endocuticle, which in some species are further subdivided into sublayers. The outermost layer, the thin epicuticle, does not contain chitin. It consists of protein and lipids and contain almost no mineral $[6,15$, 16]. Epi, exo- and endocuticle form the various skeletal elements of the animal. In isopods, the exo- and endocuticle are mineralized mainly with amorphous calcium carbonate (ACC), magnesium calcite and minor amounts of amorphous calcium phosphate (ACP) $[3,11,17]$. The structural patterns governing the mineral and organic phase vary between species [1]. Within the endo- and a proximal part of the exocuticle of isopods an about $4 \mathrm{~nm}$ thick layer of mineral surrounds each single chitin-protein fibril forming mineral tubes. In the distal part of the exocuticle, mineral fills the space between scarcely distributed thicker chitin-protein fibres [4-6].

The thin and flexible arthrodial membranes remain mostly unmineralized in isopods. In terrestrial isopods, it is likely a site of increased evaporative loss of water and a site where predators like spiders and scorpions can easily penetrate the animals armour. The structure and composition of the cuticle of the hard skeletal elements changes to that of the arthrodial membrane in a cuticle edge region. These edge regions are of particular interest. They must ensure prevention of cuticle failure at the transition to the arthrodial membrane caused by frequent relative movements between the soft and hard cuticle. Furthermore, they must be suitable to protect the arthrodial membrane from the access of the chelicera of spiders or the aculeus (stinger) of scorpions and may even reduce water loss through the arthrodial membrane by being hidden underneath the protective skeletal elements.

The main load bearing skeletal elements in isopods are the tergites. These are the dorsal sclerites (tergites) of the first 7 pereomeres (2th-8th thoracal segments) and of 5 dorsal segments of the pleon (Fig. 1). In Hemilepistus reaumuri

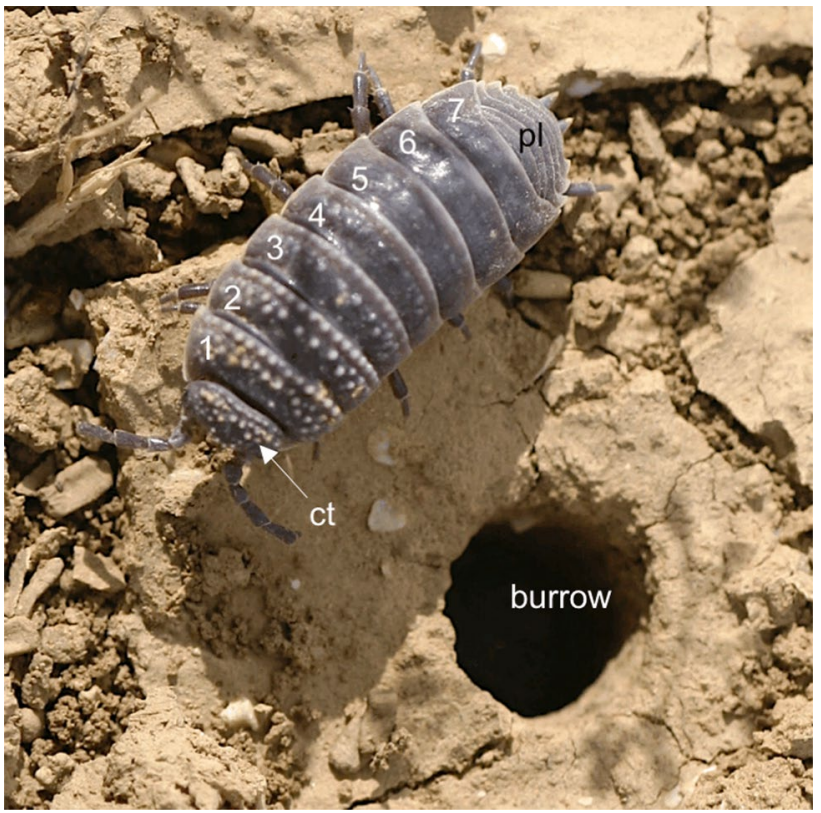

Fig. 1 The desert isopod Hemilepistus reaumuri next to its burrow. The anterior four tergites of the pereomere (numbers 1-4) are distinct from the three posterior ones (numbers 5-6) by carrying large cuticle tubercles; ct, cephalothorax, pl, pleon

(Fig. 1), the cephalothorax and the first four tergites carry large tubercles (Fig. 1), which we have studied previously [18]. The dorsal cuticle consists of the common principal cuticle layers of isopods. The exocuticle is mineralized with calcite, a distal region of the endocuticle contains both calcite and ACC, and the remaining major proximal part of the endocuticle contains ACC and small amounts of ACP [18]. The region between the tergite edges and the arthrodial membranes connecting neighbouring tergites has been studied in the terrestrial isopod Tylos europaeus [19]. T. europaeus lives at sandy beaches and burrows into the sand, in which it spends the daytime, having ready access to seawater. Upon predation, it can roll itself into a sphere to hide the soft ventral side of its body, relying on its very thick and tough cuticle [20]. In the rolled-up posture all arthrodial membranes between tergites are hidden underneath the tergite due to ventral cuticle regions at the posterior edge of the tergites (see Fig. 11 a of the Discussion). In the present study, we investigate the regions between the tergite edges and the arthrodial membrane in $H$. reaumuri, a species whose habitat and behaviour are the exact opposite of those of T. europaeus. H. reaumuri lives in semi-arid deserts and has developed a social behaviour that helps to nurture the offspring $[21,22]$. To survive the dry and hot conditions during the day in summer, it digs deep burrows that provide humid conditions. They form monogamous pairs and raise and feed their offspring within the burrows. One of the parents guards the burrow entrance with its cephalothorax and 
the anterior tergites to prevent other desert isopods to enter and to maintain the humid conditions in the burrow, while the other forages for food. In contrast to T. europaeus, $H$. reaumuri cannot roll into a sphere to protect its ventral side of the body [20]. Nevertheless, it is in some regions the most abundant animal in its habitat [22].

Comparison of the edge regions between the tergites of T. europaeus with those of $H$. reaumuri will help to discriminate between species-specific adaptations in structure and composition and their relation to the animal's habitat and behaviour on the one hand, and general characteristics of the edge regions on the other. The aim of the present study is to extend our knowledge on the morphology, ultrastructure, mineral distribution and composition as well as the crystallographic properties of the distal calcite layer and the local mechanical properties within the edge regions. In particular, we were interested in adaptations to burrowing, high daytime temperatures and predation avoidance. We analysed the edge regions using transmission electron microscopy (TEM), field-emission scanning electron microscopy (FE-SEM), electron probe X-ray microanalysis (EPMA), backscatter diffraction analysis (EBSD) and nanoindentation experiments. Comparison of the results with the anterior and posterior edge regions of tergites in T. europaeus reveals specific adaptations to the desert habitat of $H$. reaumuri, but also provides valuable information about features that appear to be common within edge regions in the exoskeleton of terrestrial isopods.

\section{Materials and methods}

\subsection{Preparation of tergites}

Twelve individuals of $H$. reaumurii (Milne-Edwards, 1840) were collected in semi-arid regions near Kairouan, Tunisia. Samples were dissected in $100 \%$ methanol. The pereonite tergites 2 (anterior) and 6 (posterior) were dissected and most of the soft tissue was removed. Samples were washed in bi-distilled water for 1-2 $\mathrm{s}$ to remove body fluids and then immediately washed in $100 \%$ methanol for about $5 \mathrm{~s}$ before being immersed in fresh $100 \%$ methanol for at least $10 \mathrm{~min}$. The tergites were air-dried overnight on filter paper and stored at $-20{ }^{\circ} \mathrm{C}$ until further use.

\subsection{Scanning (SEM) and field emission scanning electron microscopy (FE-SEM)}

For SEM micrographs of whole air dried anterior and posterior tergites, samples were mounted on aluminium studs and sputter-coated with $20 \mathrm{~nm}$ gold/palladium (MED 010, Balzers Union). Micrographs were recorded with a DSM
962 SEM (Zeiss, Germany) at an acceleration voltage of 4 or $10 \mathrm{kV}$.

For SEM analysis knife-polished, or knife polished and etched tergite pieces were used. For knife polishing, samples were first sectioned with razor blades, followed by cutting a defined planar surface with a histo-type diamond knife (Diatome, Switzerland) mounted to an Ultracut ultramicrotome (Leica, Germany). To obtain high surface qualities, the section thickness was successively decreased from 90 over 70 , $40,20,10$ to $5 \mathrm{~nm}$ at least 15 times for each step [23]. To reveal the organization of organic fibres by gentle removal of the surrounding mineral phase some of these polished samples were incubated for $20 \mathrm{~s}$ in a solution of $\mathrm{pH} 6.5$ containing $0.1 \mathrm{~mol} \mathrm{~L}^{-1}$ HEPES and $2.5 \%$ glutaraldehyde for simultaneous etching and fixation. Etched samples were washed 3 times in 100\% isopropanol for $10 \mathrm{~min}$ and critical point dried using a Bal-Tec CPD 030 [6]. Samples were rotary shadowed with 3-4 nm platinum at $45^{\circ}$ inclination using a BAF 300 (Balzers, Liechtenstein). The samples were analyzed with a Hitachi S-5200 FE-SEM or with the Zeiss DSM 962 at an acceleration voltage of $10 \mathrm{kV}$ using either a secondary electron or a backscattered electron detector.

\subsection{Transmission electron microscopy (TEM)}

For TEM, anterior and posterior tergites were decalcified and simultaneously fixed in a solution of $0.5 \mathrm{~mol} \mathrm{~L}^{-1}$ EDTA, $0.1 \mathrm{~mol} \mathrm{~L}^{-1} \mathrm{HEPES}, 2.5 \%$ glutaraldehyde and $2 \%$ paraformaldehyde at $\mathrm{pH} 7.8$ for approximately 13 to 20 days at $4{ }^{\circ} \mathrm{C}$ [6]. Then, the tergites were washed 3 times in bi-distilled water for $10 \mathrm{~min}$ each and post fixed in an aqueous solution of $1 \% \mathrm{OsO}_{4}$ and $0.8 \% \mathrm{~K}_{4}\left[\mathrm{Fe}(\mathrm{CN})_{6}\right]$ for 60 to $70 \mathrm{~min}$. The tergites were washed again 3 times with bi-distilled water, dehydrated in a graded series of isopropanol solutions and washed twice in $100 \%$ acetone. The tergite samples were embedded in EPON-resin and ultrathin sections were cut with an Ultra-type diamond knife (Diatome, Switzerland). The sections were transferred on carbon-coated formvar films on $1 \mathrm{~mm}$ copper hole-grids and stained for $10 \mathrm{~min}$ with $2 \%$ uranyl acetate and for $10 \mathrm{~s}$ with lead citrate. The samples were viewed using a Jeol-1400 TEM at $120 \mathrm{kV}$ using an Olympus digital camera $(2000 \times 2000$ pixels $)$ and the iTEM software.

\subsection{Electron probe microanalysis (EPMA)}

For EPMA, polished samples were coated with a 3-5 nm thick carbon layer. Either the FE-SEM (Hitachi S-5200) with a Phoenix (EDAX) X-ray detector system or the Zeiss DSM 962 equipped with an EDAX Falcon system were used for the analysis at acceleration voltages of $10 \mathrm{kV}$ or $20 \mathrm{kV}$. Elemental maps were recorded at a pixel resolution of $512 \times 400$ and a dwell time of $200 \mu$ s per pixel leading to 88 full scans 
per hour. A full spectrum was recorded and stored for every pixel (spectral mapping). At each scan, spectra were integrated for every pixel allowing for standardless backgroundcorrected calculation of atomic ratios for selected regions within the maps and the creation of background corrected maps of the element distribution using the GENESIS software (EDAX).

\subsection{Electron backscatter diffraction (EBSD)}

EBSD measurements were carried out on diamond knife polished surfaces as described above for FE-SEM samples. Samples were rotary coated with $4-6 \mathrm{~nm}$ of carbon. The measurements were performed with a Hitachi SU 5000 FESEM, equipped with an Oxford Instruments EBSD detector. The FE-SEM was operated at $20 \mathrm{kV}$. Obtained diffraction data were evaluated with the CHANNEL 5 HKL software. We present orientation information with colour-coded EBSD maps and corresponding pole figures. Similar colours in the map indicate similar crystal orientations, different colours highlight differences in crystal orientation. The pole figures show either individual data points or density distributions of c- and a-axes poles. In addition, we show grey-scaled band contrast measurement images. Band contrast visualizes the signal strength of the diffraction pattern in each measurement point. The strength of the EBSD signal is high when a crystal is detected (depicted in the map with light grey), whereas it is weak or absent when a polymer is scanned or when minute crystallites overlap, so that the orientation cannot be resolved automatically (dark/black). For further information see [24] and references therein.

When in a pure calcite crystal that is grown from solution calcite crystallites are not misoriented to each other, then this calcite crystal is a single crystal. However, are slight misorientations between crystallites present, then we regard this crystal as an 'almost single crystal'. For further information see [25].

\subsection{Nanoindentation testing}

A Hysitron TriboIndenter TI 900 testing device equipped with a force transducer mounted to a top-down closed-loop scanner was used to measure local mechanical properties of different cuticle regions. The measurements were carried out on cuticle pieces where the regions of interest were polished using a diamond knife (Diatome) mounted to a Leica Ultracut ultramicrotome to obtain a surface quality suitable for the experiments. The measurements were carried out using a pyramidal Berkovich indenter tip (Hysitron, Ti 39-01) with a tip radius of $50 \mathrm{~nm}$. Samples were first scanned in imaging mode followed by determination of rectangular indent patterns large enough to cover each area of interest. The horizontal and vertical spacing between individual indents was set to $5 \mu \mathrm{m}$ to avoid overlap of the deformed zones around each indent. We operated the force transducer in displacement control mode with a fixed indentation depth of $300 \mathrm{~nm}$. The load function was trapezoidal with $5 \mathrm{~s}$ for loading, $20 \mathrm{~s}$ holding time and $5 \mathrm{~s}$ for unloading. All force-displacement curves were calibrated using an area function determined on PMMA (1.7 nm to $365.4 \mathrm{~nm}$ ). The reduced elastic modulus $\left(E_{\text {red }}\right)$ and the hardness $(\mathrm{H})$ values were calculated with the TriboScan (Hysitron) software package as described in [26]. Although being aware that hydration affects the mechanical properties of cuticle to different extents in mineralized and unmineralized regions, we nevertheless used dry samples for nanoindentation. This was due to the time required to polish the sample surfaces and the small indentation depth, which make maintaining the original hydration state of the sample unfeasible. Likewise, it was not reasonable to use rehydrated samples, since superficial dissolution of mineral phase components and overhydration of chitin and proteins could not be excluded. It must therefore be noted that the nanoindentation results obtained do not precisely reflect the mechanical properties of cuticle in the native hydrated state.

\subsection{Statistical analysis}

Statistical analysis was performed with the GraphPad Prism software version 6.00 for Mac (California USA, www.graph pad.com). Means and standard deviations were calculated. For the nanoindentation tests, two-way ANOVA followed by Sidak's multiple comparisons tests were used to assign significant differences between the means of $E_{\mathrm{red}}$ and $\mathrm{H}$ of selected regions and layers of the ventral cuticle. An oneway ANOVA was used to test significant differences between cuticle layers in the ventral cuticle of the posterior tergite.

\section{Results}

All images of sections and polished planes are oriented in a way that the dorsal side of the animal is oriented upwards. Schemes in the figures provide the sites within the cuticle presented.

\subsection{The morphology of the tergite edge regions}

Figure 2 shows SEM micrographs and schemes of sagittal sections of an anterior (Fig. 2a, c-f) and a posterior (Fig. 2b, g) tergite. The tergites have distinct anterior and posterior parts. The posterior parts carry many scales and sensilla. In addition, the posterior parts of the first four pereon tergites carry large tubercles (Fig. 2a). The anterior part is hidden underneath the posterior edge of the preceding tergite. It has a smooth surface devoid of large scales and tubercles. Its length is about $250 \mu \mathrm{m}$, 

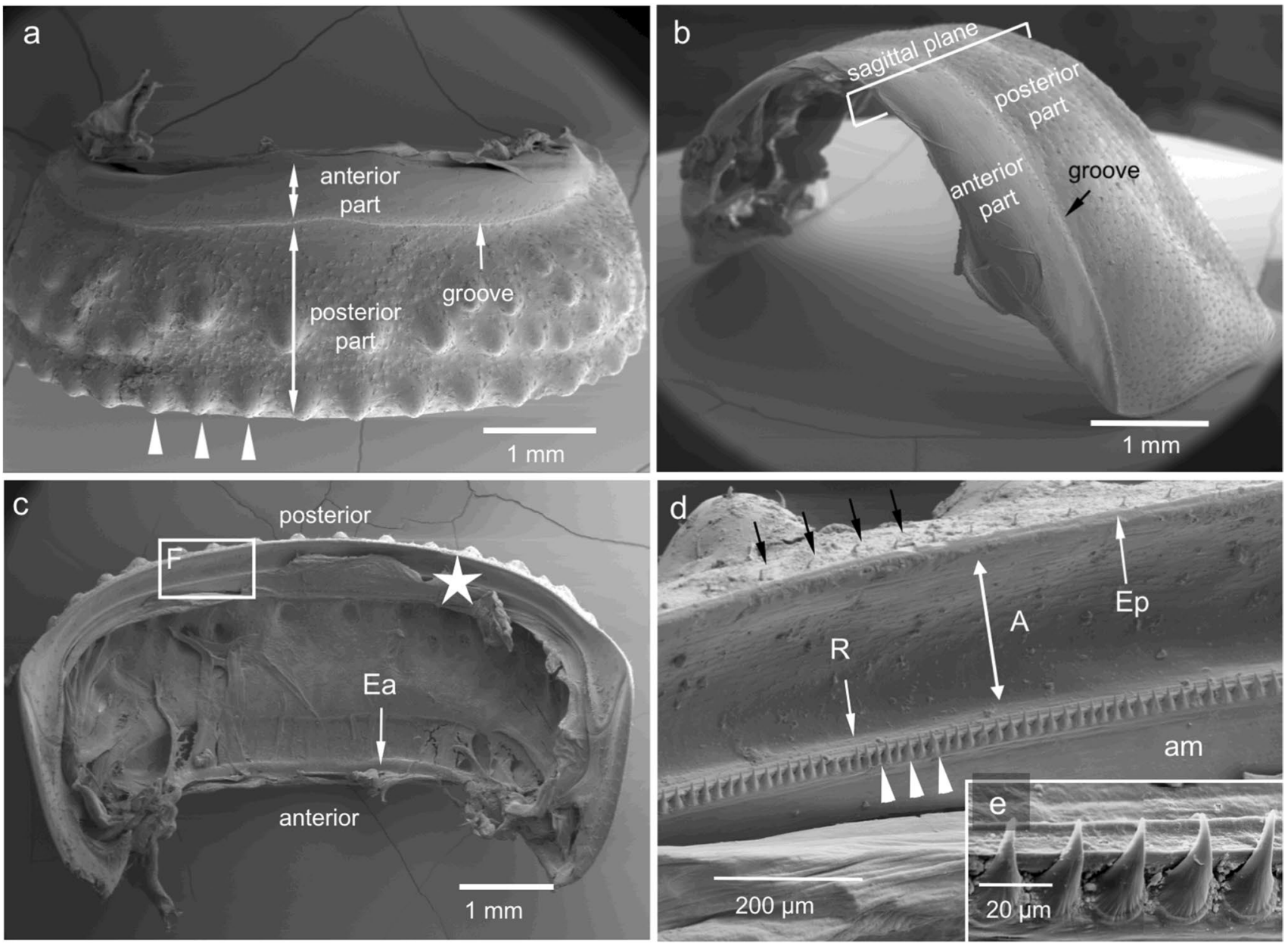

$f \quad$ anterior tergite

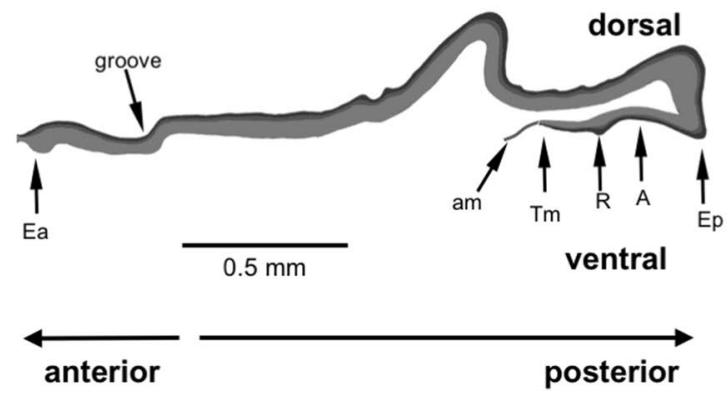

Fig. 2 SEM micrographs and schematic longitudinal profiles of tergites of Hemilepistus reaumuri. a, b Dorsal views of an anterior (a) and a lateral view of a posterior (b) tergite. Note the groove demarking an anterior and a posterior part indicated on the micrographs. c-e Ventral views of an anterior tergite. c Overview, showing the anterior edge (Ea) at the anterior side and the ventral cuticle (asterisk) folded under the posterior edge region. $\mathbf{d}$ Detail of the posterior edge and

separated from the posterior part by a groove. In sagittal sections, it forms an arch with the convex side directed to the outward side. At the anterior edge (Ea) the cuticle thickens before the transition to the arthrodial membrane

\section{9 posterior tergite}

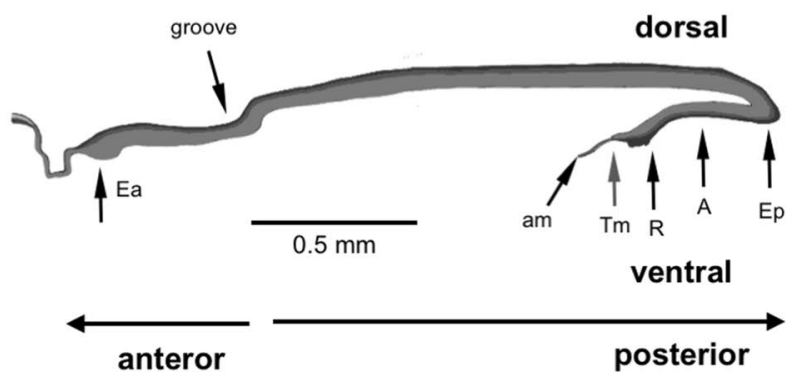

ventral cuticle as marked with a rectangle in c. Black arrows mark regular spaced sensilla dorsally of the sharp posterior edge (Ep); A, arched ventral cuticle; am, arthrodial membrane; R, ridge. White arrowheads point to regularly spaced cone-like bristles. e Detail of the bristles from (d). Schemes depicting sagittal sections through the anterior (f) and posterior (g) tergite; Tm, transition to the arthrodial membrane

(Figs. 2f, g and 3a). At the posterior edge, the tergite cuticle sharply bends down and towards the anterior direction, forming a ventral cuticle region that connects to the arthrodial membrane (Fig. 2d, f, g). The ventral cuticle 

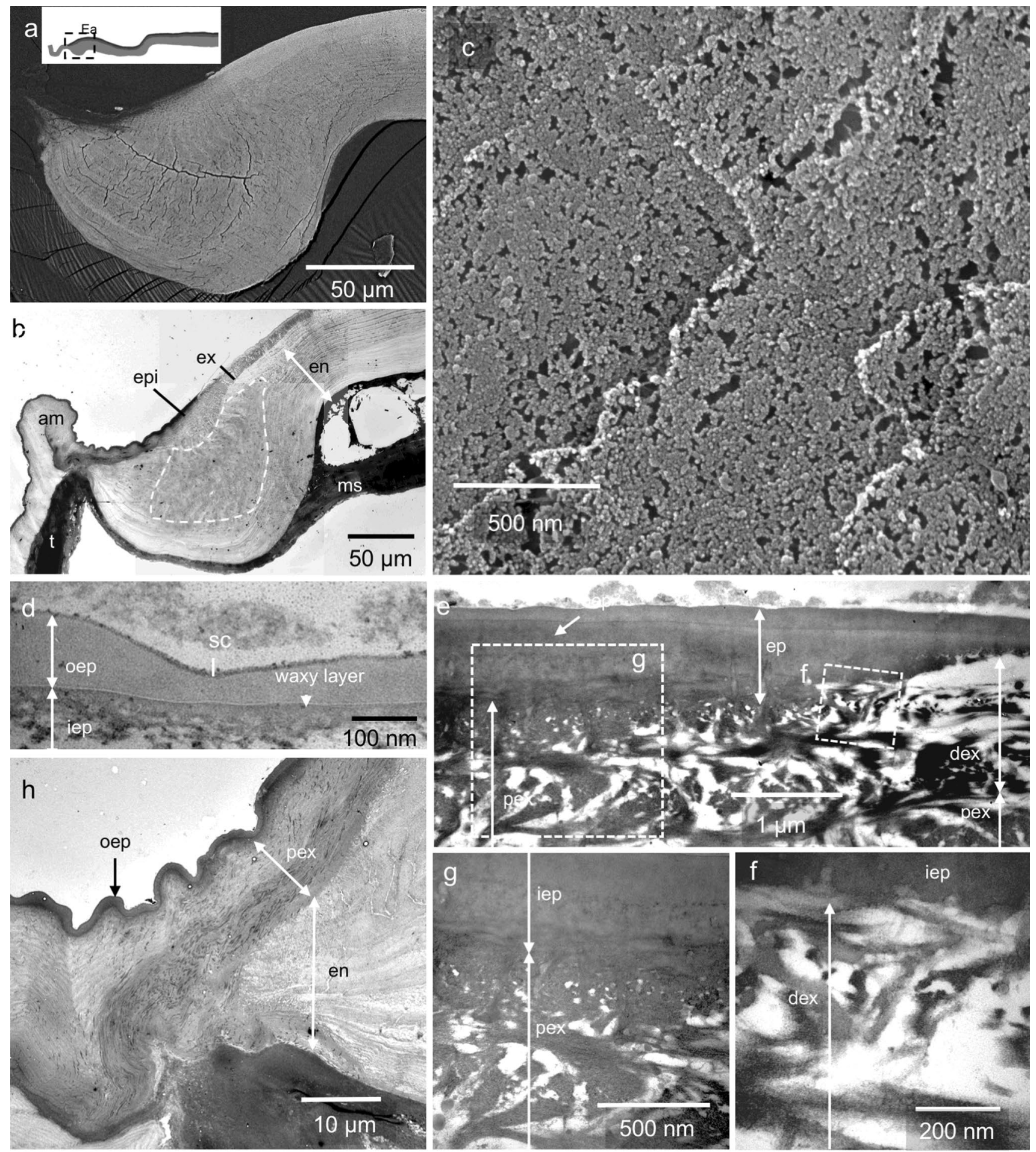

region is about $500 \mu \mathrm{m}$ long. It has four distinct regions: (1) the sharp posterior edge (Ep) where the dorsal cuticle bends down and to the anterior direction, which carries regularly spaced sensilla at the dorsal side (Fig. 2d), (2) a transition region to the arthrodial membrane (Tm), (3) a thickened ridge region (R) just before the transition to the arthrodial membrane that carries regularly spaced cone-shaped bristles (Fig. 2d-g) and (4) an about $250 \mu \mathrm{m}$ long arched region (A) between the sharp posterior edge and the thickened ridge (Fig. 2d, f, g). 
4Fig. 3 Structure of the anterior edge at a lateral side of an anterior tergite in Hemilepistus reaumuri. a BSE-SEM micrograph of a sagittally cut polished plane at the region marked by a dashed rectangle in the scheme; Ea, anterior edge. The edge forms a thick bulge. Regions with dense material appear brighter. b TEM micrograph showing the epicuticle (epi) exocuticle (ex) and endocuticle (en). A muscle (ms) attaches to the bulge; am, arthrodial membrane; $t$, tissue attached to the arthrodial membrane. The dashed outline marks the area in which protein-chitin fibrils are oriented unidirectionally. c SEM micrograph of a sagittally polished and etched surface from the region containing parallel oriented fibrils. The long axis of the fibrils point to the lateral sides of the tergite. $\mathbf{d}$ TEM micrograph of the epicuticle before the transition to the arthrodial membrane. The epicuticle consists of a surface coat (sc) an outer (oep) and an inner epicuticle (iep). A waxy layer in the outer epicuticle can appear as a dense line. e-g TEM micrographs of the epicuticle and part of the exocuticle at the transition to the arthrodial membrane. e Overview depicting the region of epicuticle (ep) thickening. The darker distal exocuticle (dex) disappears and epicuticle and proximal exocuticle (pex) become thicker. $\mathbf{f}$ Distal exocuticle at the region marked by the small dashed rectangle (f) in figure e. g Proximal exocuticle and iep epicuticle at the region marked by the large rectangle $(\mathbf{g})$ in figure e showing that the distal exocuticle is lacking. $\mathbf{h}$ TEM micrograph showing details at the very tip of the transition

\subsection{Ultrastructure and mineral distribution in the anterior edge region}

Figures 3 and 4a show the ultrastructure and the mineral distribution within the anterior edge region of an anterior tergite (Ea in Fig. 2f). The dorsal cuticle before the anterior edge resembles that of the general dorsal cuticle that has been described previously $[18,27]$. Within back-scatter electron SEM micrographs, the distal layers containing calcite appear denser than those mineralized with ACC (Figs. 3a and $4 \mathrm{a}$ ). The anterior edge is characterized by the formation of a cuticular bulge and the disappearance of the denser mineral layers (Fig. 3a, b). The thickness of the bulge is particularly large near the lateral sides of the tergites that serve as muscle attachment sites (Fig. 3b). In the middle regions, where no muscles attach, the bulge is thinner (Fig. 4a). Thickening of the cuticle forming the cuticle bulge is caused by a thickening of the proximal exocuticle and 3-4 distal stacks of the endocuticle (Fig. 3a, b). Before the stacks of the endocuticle become thicker, they fuse forming a single stack. The chitin-protein fibrils within these fused stacks run in parallel with the longitudinal axes pointing to the lateral sides (Fig. 3c). Before the transition to the arthrodial membrane the epicuticle increases in thickness from $100 \mathrm{~nm}$ to about $1 \mu \mathrm{m}$ and the distal exocuticle disappears (Figs. 3d-g). Thickening of the epicuticle is brought about by an increase of the inner epicuticle, while the outer epicuticle remains thin. At the transition to the arthrodial membrane, the stack containing only parallel fibres forms several stacks again (Fig. 3a, b). Here the stacks of the exocuticle stain denser (Fig. 3b). The exocuticle and stacks of the endocuticle continue into the arthrodial membrane (Fig. 3h), which is devoid of mineral (Fig. 3a). Elemental maps and EDX spectra of selected regions show that the phosphorus content increases towards the transition to the arthrodial membrane, whereas the distribution of magnesium remains more or less constant (Fig. 4a, b).

In the anterior edge region of posterior tergites (Ea in Figs. $2 \mathrm{~g}$ and $4 \mathrm{c}$ ) a similar increase in the phosphorus content was observed at the transition to the arthrodial membrane. In contrast to the anterior tergites, we found that the proximal half of the arthrodial membrane still contains mineral near the edge with a high phosphorus content (Fig. 4c-e).

\subsection{Ultrastructure and mineral distribution in the posterior edge and ventral cuticle of the anterior tergite}

Figure 5 shows an overview of the posterior edge (Fig. 5a) as well as details of the sharp posterior edge (Ep in Fig. 2f; Fig. 5b-e) and of the ventral arc-shaped cuticle (A in Fig. 2f; Fig. 5f). At the region of the edge the distal layer of dense mineralization increases several times (Fig. 5a) and the overall thickness of the cuticle increases somewhat. The epicuticle is thin (Fig. 5c). It consists of an about $30 \mathrm{~nm}$ thick outer epicuticle having a densely stained thin layer in the middle. This dense layer is likely a waxy layer [28]. The densely stained inner epicuticle is about $100 \mathrm{~nm}$ thick. At the sharp posterior edge, the distal exocuticle, which is thin in the dorsal and ventral cuticle, increases in thickness to up to $10 \mu \mathrm{m}$ (Fig. 5d). The distal exocuticle is characterized by fibres consisting of several chitin fibrils embedded in a densely stained, likely proteinaceous matrix (Fig. 5e). The proximal exocuticle almost doubles its thickness and the thickness of distal stacks of the endocuticle also increase (Fig. 5b, d). About $100 \mu \mathrm{m}$ before the dorsal cuticle bends downwards, 3 stacks of the more proximal endocuticle fuse to a single about $20 \mu \mathrm{m}$ thick stack (Fig. 5b). The protein-chitin fibrils within this stack are parallel to one another with their long axis oriented to the lateral sides of the tergite. At the curve the fibril orientations within the stacks of the endocuticle are distorted (Fig. 5b, d). At the ventral side, more proximal stacks fuse with the stack of parallel fibrils (Fig. 5b). The arced ventral cuticle between the edge and the ridge region is thinner than the dorsal tergite cuticle (Fig. 5a). Here, stacks of the proximal endocuticle form a single stack with fibrils oriented in parallel (Fig. 5f).

About $150 \mu \mathrm{m}$ before the transition to the arthrodial membrane the cuticle forms a ridge ( $\mathrm{R}$ in Figs. 2f; 5a and $6 \mathrm{a}-\mathrm{c})$ that is characterized by the before mentioned cone like hairs (Fig. 1d, e). At the ridge, the cuticle thickness increases from about $50 \mu \mathrm{m}$ at the arced ventral cuticle to $80 \mu \mathrm{m}$ forming two ribs (Fig. 6b, c). The outer epicuticle increases from 30 to $100 \mathrm{~nm}$ while the inner epicuticle remains unchanged in comparison to the arched ventral 
Fig. 4 The composition of the anterior edge in an anterior (a, b) and posterior $\mathbf{c}, \mathbf{d})$ tergite. $\mathbf{a}$ Backscattered electron micrograph (BSE-SEM) and elemental spectral maps for calcium, phosphorus and magnesium recorded on a polished sample cut sagittally through a median site. b EDX spectra of the entire cuticle and of the regions 1-3 marked with rectangles in the BSE-SEM micrograph in a. Note the high phosphorus peak at the transition to the arthrodial membrane (rectangle 3 in A). The molar ratios for $\mathrm{Mg}$, $\mathrm{P}$ and $\mathrm{Ca}$ are provided in the diagram. c BSE-SEM micrograph and elemental spectral maps for calcium, phosphorus and magnesium of a polished sample cut sagittally through a median site. d EDX spectra of the cuticle and of the regions 1 and 2 marked with rectangles in the BSE-SEM micrograph in c. Note the high phosphorus peak at the transition to the arthrodial membrane (rectangle 2 in c). e EDX spectra of the membranous layer of the region 3 marked with a dashed line in the phosphorus map in c. The molar ratios for $\mathrm{Mg}, \mathrm{P}$ and $\mathrm{Ca}$ are provided for the three regions
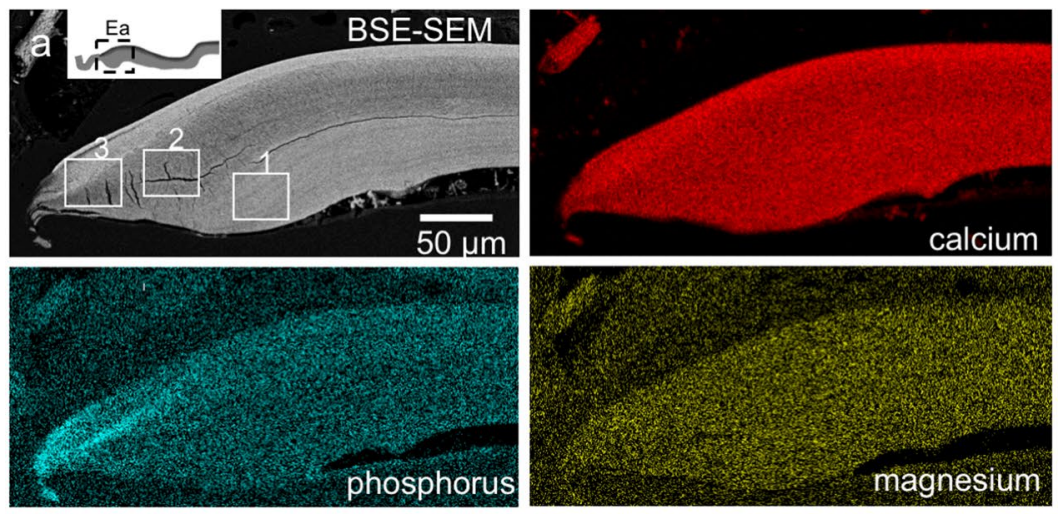

b

$\mathrm{Mg} / \mathrm{P}$

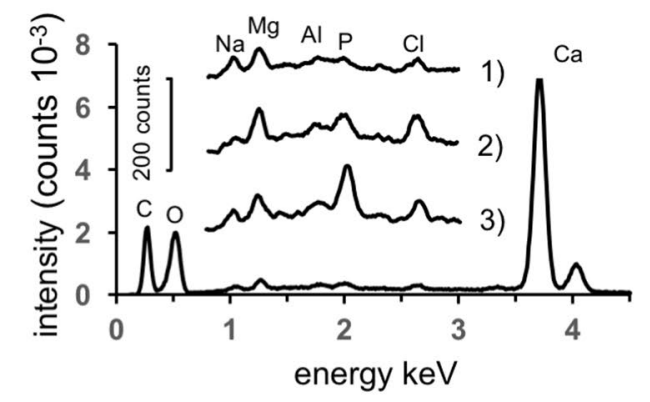

1) $5.6 / 1.5$

2) $6.4 / 2.3$

3) $5.7 / 7,1$
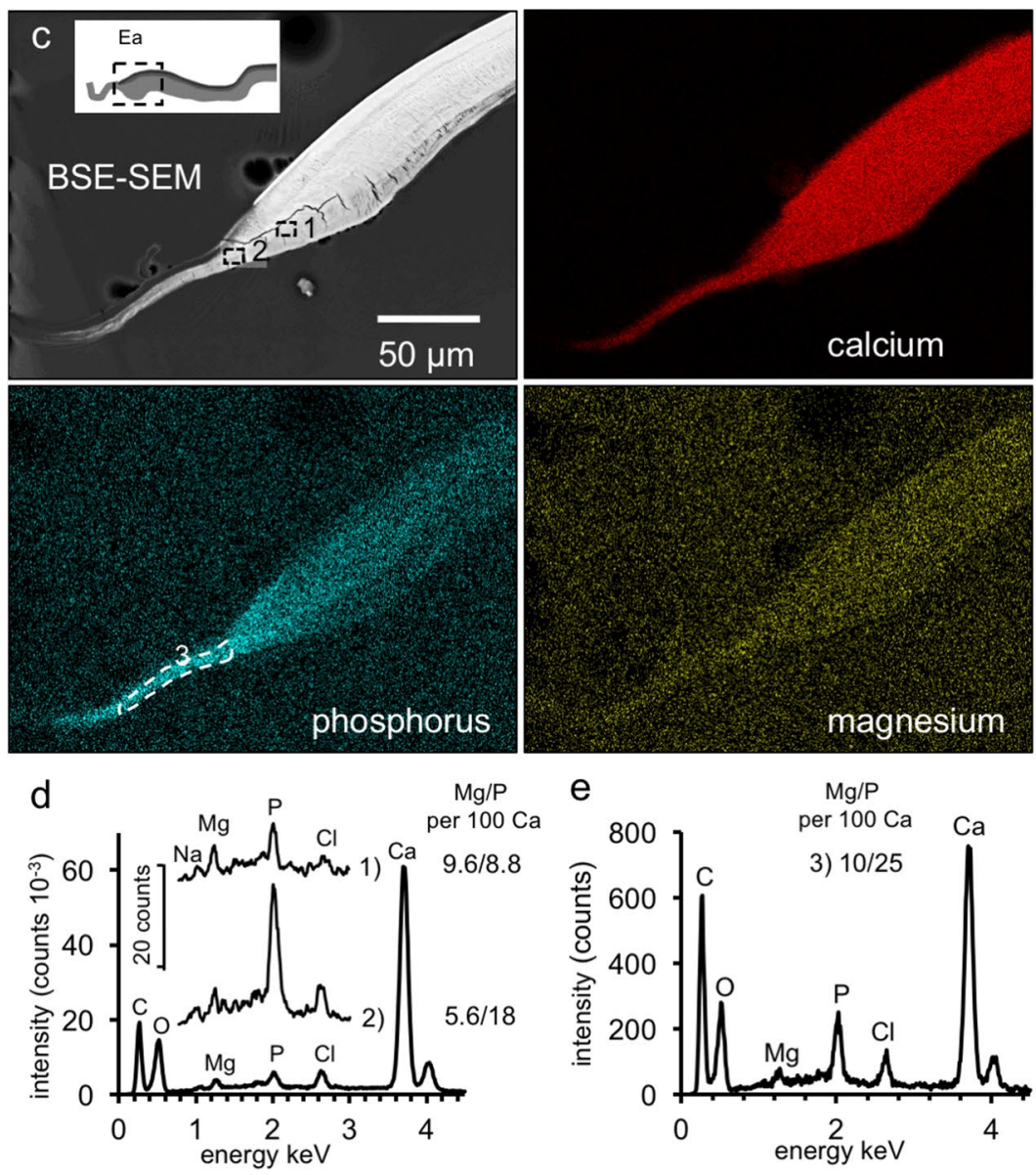

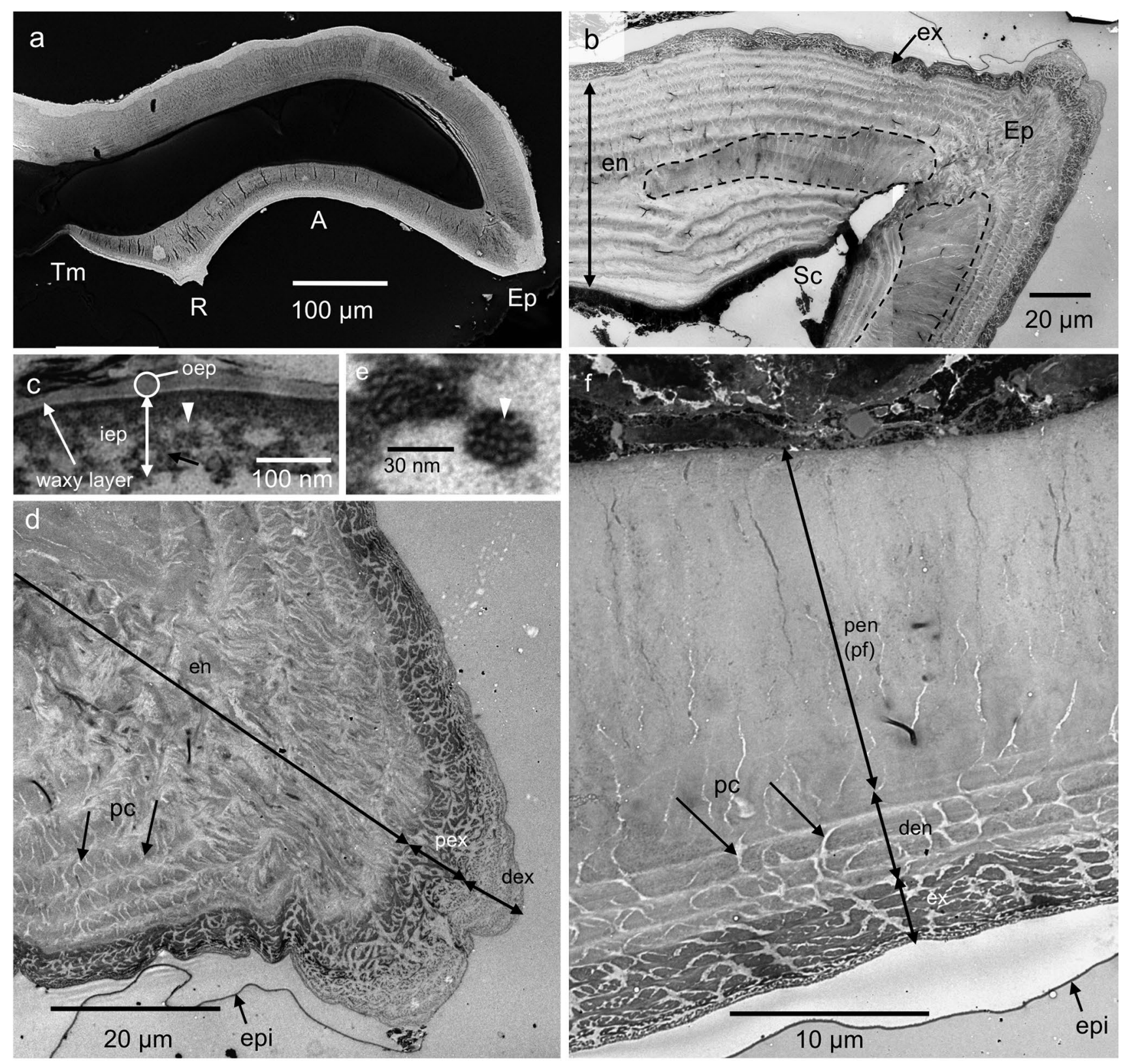

Fig. 5 Structure of the posterior edge of an anterior tergite in Hemilepistus reaumuri. a BSE SEM micrograph of a sagittally cut and polished plane; A, arched ventral cuticle between the sharp posterior edge (Ep) and the ridge (R); Tm, transition to the arthrodial membrane. Regions with dense material appear brighter. b-f TEM micrographs. b The region around Ep showing the exo- (ex) and endocuticle (en); Sc channel for a sensillum through Ep. The dashed outline marks the area in which protein-chitin fibrils are oriented in parallel. c TEM micrograph of the epicuticle consisting of a an outer (oep) and an inner epicuticle (iep). A waxy layer in the outer epicuticle can

cuticle. The outer epicuticle consists of a thin dense and thin bright layer at its distal and proximal side enclosing a thick dense layer (Fig. 6d). At the ridge, the proximal and in particular the distal exocuticle become thicker forming the two ribs (Fig. 6b, c, e). The endocuticle is subdivided appears as a dense line. d Detail of the sharp posterior edge, showing an increase of the distal (dex) and proximal exocuticle (pex) and of the endocuticle; pc, pore canals. The epicuticle has detached from dex. e detail from dex showing fibres consisting of a number of chitin fibrils (arrowhead) within a likely proteinaceous matrix. f Detail from the ventral arched cuticle between the sharp posterior edge and the ridge, marked with A in Figure a. The proximal endocuticle (pen) forms a single stack of parallel chitin-protein fibrils (pf); den; distal endocuticle. The arrows point to pore canals

into an about $12 \mu \mathrm{m}$ thick distal endocuticle and a $40 \mu \mathrm{m}$ thick proximal endocuticle. The proximal endocuticle consists mainly of a single stack of parallel fibrils (Fig. 6b) that spans from the edge to the transition to the arthrodial membrane (Figs. 5b, f and 6b, f). At the anterior side of 

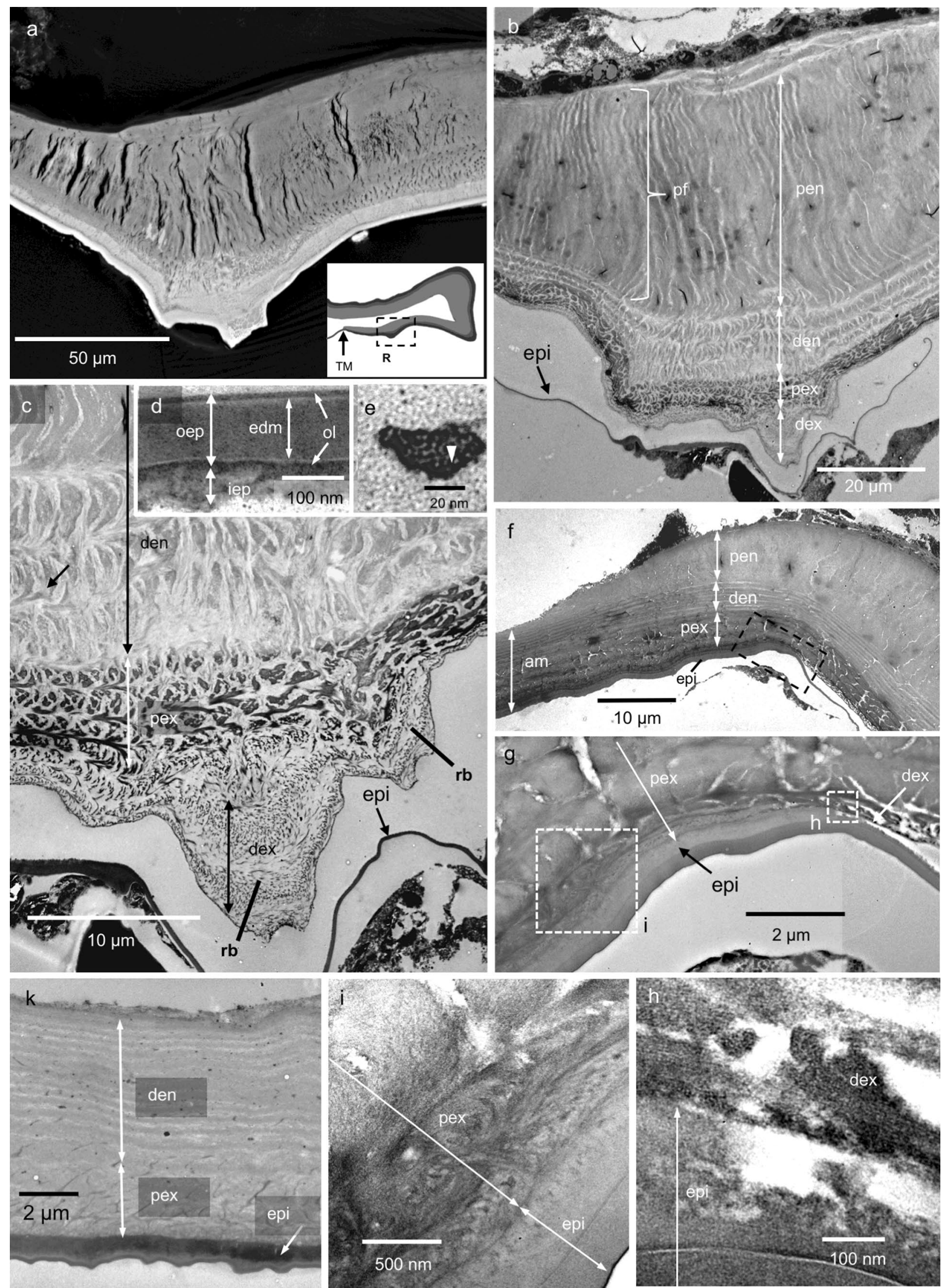
4Fig. 6 Structure of the ridge (R) within the ventral cuticle and the transition to the arthrodial membrane (TM) at the posterior side of an anterior tergite of Hemilepistus reaumuri. a BSE-SEM micrograph of a sagittally cut and polished plane at the region marked by a dashed rectangle in the scheme. Regions with dense material appear brighter. b-e TEM micrographs recorded from the ridge. b Overview showing the detached epicuticle (epi), the distal (dex) and proximal exocuticle (pex), the distal (den) and the proximal endocuticle (pen); pf, layer of unidirectionally oriented fibrils. c Detail of the ridge showing the changes in thickness of dex and pex at the ribs (rb). d Detail of the epicuticle with a thick outer epicuticle (oep) and a thin inner one (iep); edm electron dense layer in the middle; ol, outer dense and bright layers. e detail from the distal exocuticle showing a fibre consisting of chitin fibrils within a proteinaceous matrix. $\mathbf{f}-\mathbf{i}$ TEM micrographs of the transition to the arthrodial membrane. f Overview, showing that the proximal endocuticle of fibrils with parallel orientation does not enter the arthrodial membrane (am). g Detail from the region marked with a dashed rectangle in figure $\mathbf{f}$ depicting the region of epicuticle thickening. $\mathbf{h}$ Distal exocuticle at the region marked by the small dashed rectangle (h) in figure g. i Proximal exocuticle and epicuticle at the region marked by the large rectangle (i) in figure g, showing that the distal exocuticle is lacking. $\mathbf{k}$ TEM micrograph of the arthrodial membrane between tergites, consisting of the epicuticle (epi), the proximal exocuticle (pex) and the distal endocuticle (den) of the tergite cuticle

the ridge the cuticle bends towards the dorsal side and its thickness decreases to about $40 \mu \mathrm{m}$ and then continuously decreases in thickness by a factor of four. Just before the cuticle connects to the arthrodial membrane it bends to the ventral side (Figs. 5a and 6f). Here the distal exocuticle disappears and the epicuticle increases in thickness to about $1 \mu \mathrm{m}$ (Fig. $6 \mathrm{f}-\mathrm{i}$ ). The thickness of the proximal exocuticle increases only slightly (Fig. 6f). At the transition with the arthrodial membrane (Tm in Fig. 2f) the proximal endocuticle of parallel fibres ends. The arthrodial membrane consists of the $1 \mu \mathrm{m}$ thick epicuticle, the former proximal exocuticle, and the distal endocuticle (Fig. 6f, k) The outer epicuticle of the arthrodial membrane varies in thickness between 400 and $800 \mathrm{~nm}$ (Fig. 6k). It has an outer layer that is more electron dense than its inner layer. The inner epicuticle is electron dense and 500 to $600 \mathrm{~nm}$ thick (Fig. 6k).

Elemental maps and EDX spectra of the sharp posterior edge (Fig. 7a), the ridge (Fig. 7b) and the transition to the arthrodial membrane (Fig. 7c) show that the phosphorus and calcium contents are higher within the distal layers of the cuticle. The magnesium is more or less evenly distributed at the edge (Fig. 7a). At the ridge and at the transition to the arthrodial membrane magnesium is more concentrated within the distal layer of the cuticle (Fig. 7b, c). BSE-SEM images and the element distribution maps for calcium and phosphorus show that for about $60 \mu \mathrm{m}$ the distal region of the arthrodial membrane still contains mineral (Fig. 7c). The phosphorus content is in particular high at the transition to and in the arthrodial membrane, where the phosphorus/calcium ratio rises to 0.28 .

\subsection{Patterns of crystal orientation within the calcite layer}

The high variation in calcite layer thickness, deduced from EBSD and measured with a step size of $200 \mathrm{~nm}$, is presented in Figs. 8 and S1. It varies significantly for the different cuticle regions. The calcite layer is about $2 \mu \mathrm{m}$ at the transition to the arthrodial membrane (Figs. $8 \mathrm{~b}$ and S1c), but considerably thicker at the tubercle tip (Figs. 8f, S1e and [18]), the sharp bend at the posterior edge (Figs. 8d and S1b) and at tubercle slopes (Figs. 8e and [18]). In addition, at nontubercular regions (Fig. S1d) and, especially, at tubercle tips (Fig. 8f, g) we observe a distally located dense calcite layer that is complemented proximally by patchy calcite accumulations. In contrast, close to the sharp posterior edge (Fig. $\mathrm{S} 1 \mathrm{~b})$, the ridge and at the transition to the arthrodial membrane (Figs. 8b, c and S1c) such patchy calcite incorporations are absent; only the distally developed dense calcite layer was observed.

Patterns of calcite orientation at different tergite regions are shown in Fig. 8. The calcite layer consists of large mineral units, with calcite crystallites in these being highly cooriented. This is specifically the case for the ventral cuticle, near the transition to the arthrodial membrane (Fig. 8b) and for the ridge (Fig. 8c). The mineral units at the latter two cuticle regions are almost single crystals (see the even colours in Figs. 8b, c). Two texture patterns are present within the anterior tergite of $H$. reaumuri. At tubercle tips, there is a bimodal distribution of calcite (e.g. Figure $8 \mathrm{f}$ ) with calcite c-axes being mainly within the plane of view of the sagittal cut samples. In the ventral cuticle (ridge, transition to arthrodial membrane) mineral units become single crystalline and consist of calcite crystals that have their c-axes oriented perpendicular to the plane of view (Figs. 8b, c). Thus, from tubercle tip to the transition to the arthrodial membrane calcite orientation rotates by 90 degree.

\subsection{Ultrastructure of the posterior edge and ventral cuticle in posterior tergites}

The overall structure of the posterior edge and ventral cuticle in posterior tergites is very similar to those in the anterior tergites, solely the dorsal and ventral cuticle near the sharp edge (Ep in Fig. 2g) are about one-third thinner (Fig. 9a-c). This difference is mostly due to a thinner proximal endocuticle. The thickness of the distal and proximal exocuticle at the sharp edge of $7 \mu \mathrm{m}$ each resembles that of the anterior tergite (Fig. 9d, e). The largest difference occurs within the ridge region ( $\mathrm{R}$ in Fig. $2 \mathrm{~g}$ ), which is two times longer than in the anterior tergite (Fig. 9f, g). Furthermore, the ridge carries many long scales at the anterior sides (Fig. 9f) and the transition to arthrodial membrane starts immediately anterior to the ridge (Fig. S2), resulting in a longer arched 

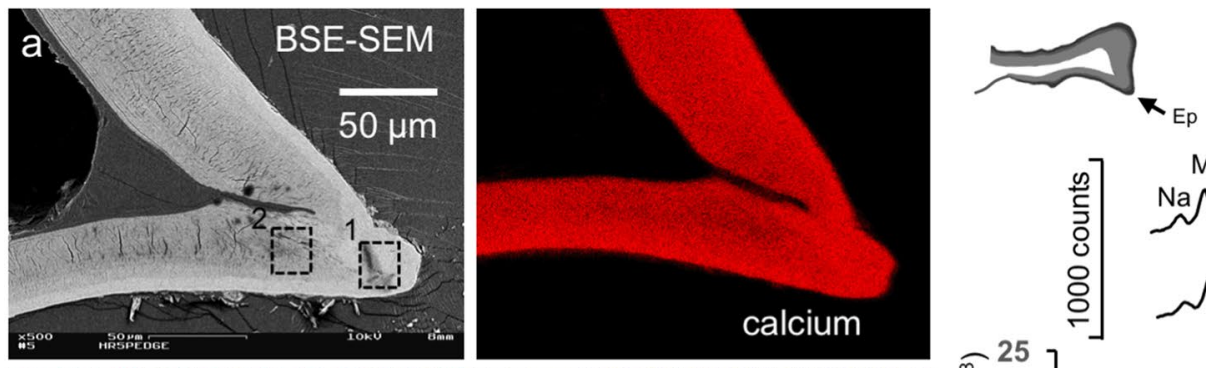

$\mathrm{Mg} / \mathrm{P}$
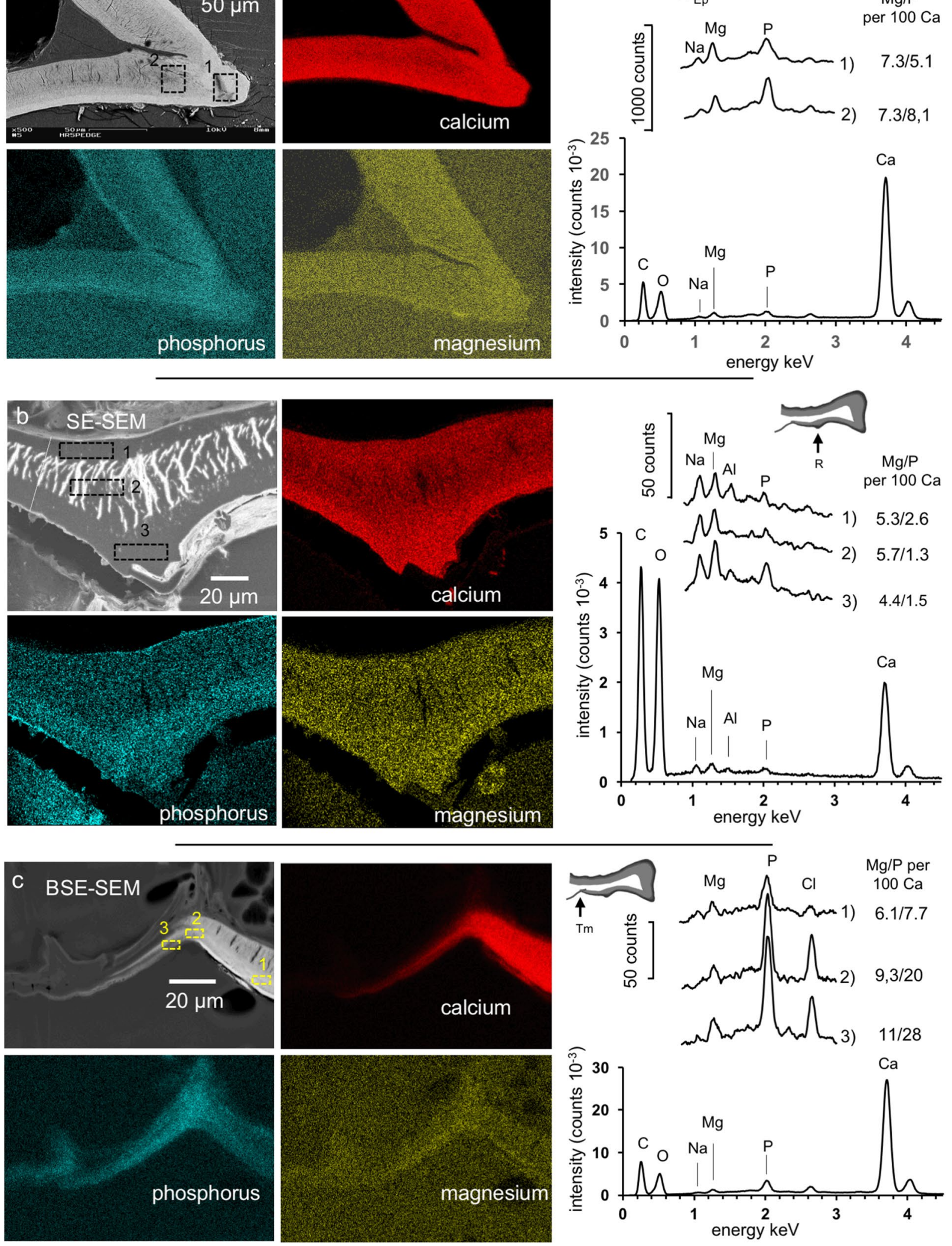
4Fig. 7 The composition of the posterior edge and ventral cuticle in an anterior tergite. The images show BSE-SEM or SE-SEM micrographs and elemental spectral maps for calcium, phosphorus and magnesium of sagittally cut and polished samples. EDX spectra of the entire cuticle and of the regions marked with rectangles in the SEM micrograph are provided on the left side of the EDX-maps. The molar ratios for $\mathrm{Mg}, \mathrm{P}$ and $\mathrm{Ca}$ are provided in the diagrams. a The edge. $\mathbf{b}$ The ridge of the ventral cuticle. $\mathbf{c}$ The transition to the arthrodial membrane. Note the high phosphorus peak at the transition to the arthrodial membrane (rectangle 2 in c) and within the arthrodial membrane (rectangle 3 in c)

ventral cuticle region. The mineral distribution within the ridge is also similar to that of the anterior tergite with a thick distal layer of dense mineral and of low density within the proximal endocuticle (Fig. 9g). Elemental maps of the ventral cuticle show that within the distal region the calcium content is higher than in the proximal endocuticle (Fig. S2). Within the ridge magnesium is also higher in distal layers (Fig. S2f). At the transition to the arthrodial membrane the phosphorus concentration is particularly high (Fig. S2b, e).

\subsection{Local mechanical properties of the ventral cuticle region}

Nanoindentation experiments have been performed in five different regions of the ventral cuticle of anterior tergites (Fig. 10): The arthrodial membrane (region A), the transition to the arthrodial membrane (region $\mathrm{B}$ ), the region posterior of the arthrodial membrane (region $\mathrm{C}$ ), the region anterior to the ridge (region $\mathrm{D}$ ) and the region posterior to the ridge (region E). It was not possible to obtain results from the epicuticle of regions B to E since they were too thin for reliable measurements. For the relatively thick epicuticle in the arthrodial membrane two indents yielded a reduced elastic modulus $(\mathrm{Er})$ and a hardness $(\mathrm{H})$ for the epicuticle of $6.7 \pm 0.5 \mathrm{GPa}$ and $0.26 \pm 0.07 \mathrm{GPa}$, respectively. Within the exo/endocuticle of the arthrodial membrane Er was $14.5 \pm 2.6$ (14) GPa and H $0.52 \pm 0.12$ (14) GPa, about half the values measured in region $B$.

At region $\mathrm{B}$, the mean Er values for the exocuticle, stacked distal exocuticle and the endocuticle with its parallel fibrils were between 28.2 and $29.2 \mathrm{GPa}$ and not significantly different from each other (Fig. 10). The mean value for $\mathrm{H}$ was significantly higher in the exocuticle than in the two endocuticular layers. In the regions $\mathrm{C}, \mathrm{D}$ and $\mathrm{E}$, the mean values for $\mathrm{Er}$ and $\mathrm{H}$ within the exocuticle were between 34.5 and $38.4 \mathrm{GPa}$ and between 2.1 and $2.7 \mathrm{GPa}$, respectively. Both, Er and $\mathrm{H}$ were significantly higher within the exocuticle than in the stacked distal endocuticle. Within the endocuticle with parallel fibrils of the regions $\mathrm{C}, \mathrm{D}$ and $\mathrm{E}$ the mean values for Er range between 31.1 and $33.8 \mathrm{GPa}$. They are higher than in the distal endocuticle with stacks; however, this is only significant for the regions D and E. They are also higher than the values of Er of the stacked proximal endocuticle obtained from the regions $\mathrm{C}$ and $\mathrm{D}$. The mean value for $\mathrm{H}$ in the endocuticle with parallel fibres range between 1.1 and 1.3, and were significantly higher than in the distal endocuticle with stacks for the region E only.

When comparing mean values for Er between the regions B-E we found no significant differences for the stacked distal exocuticle. Between the regions $\mathrm{D}$ and $\mathrm{E}$, none of the layers yielded significant differences in mechanical properties. However, in the regions B, C and D the mean values for Er between neighbouring regions were always significantly lower at the anterior side for the proximal stacked endocuticle, the endocuticle with parallel fibrils and the exocuticle, (Fig. 10). For the mean values of $\mathrm{H}$, the differences between the neighbouring regions $\mathrm{B}, \mathrm{C}$ and $\mathrm{D}$ were significant for the exocuticle only. In the region $\mathrm{D}$, the mean value for $\mathrm{H}$ was also significantly lower than in the region $\mathrm{E}$, but for the endocuticle with parallel fibrils only.

Since in the posterior tergite the ridge immediately ends at the arthrodial membrane we have not discriminated different regions for the nanoindentation experiments. Er and $\mathrm{H}$ were $31.6 \pm 2.84$ (22) and $2.3 \pm 0.47$ (22) GPa within the exocuticle, $26.0 \pm 2.16$ (23) $1.1 \pm 0.26$ (23) GPa within the stacked distal exocuticle, and $27.0 \pm 2.42$ and $1.0 \pm 0.22$ (35) GPa within the endocuticle with parallel fibrils, respectively (values are given as mean \pm SD (number of indents)). The values for Er and $\mathrm{H}$ within the exocuticle were significantly higher than in the endocuticular layers.

\section{Discussion}

The present study provides the first thorough investigation of the cuticle at the anterior and posterior edge regions of tergites which connects them with the arthrodial membrane in the desert isopod H. reaumuri. The results enable us for the first time to compare this isopod's cuticle structures with corresponding structures in tergites of the beach isopod Tylos europaeus [19] and with the transition to the arthrodial membrane in the joint cuticle of the mesic isopod Porcellio scaber [5]. A previous study on the dorsal tergite cuticle of $H$. reaumuri [18] provides further clues to understand the functionalization of cuticle connecting rigid skeletal elements with the soft and flexible cuticle membranes.

\subsection{Functional morphology of the edges and ventral cuticle}

The morphology of the anterior edge region near the median plane of the tergite is very similar to that in T. europaeus. At lateral planes, however, where the muscle attachment sites are located they are quite distinct. In T. europaeus, the edge forms a hairpin bend serving as an apodeme for the lateral 

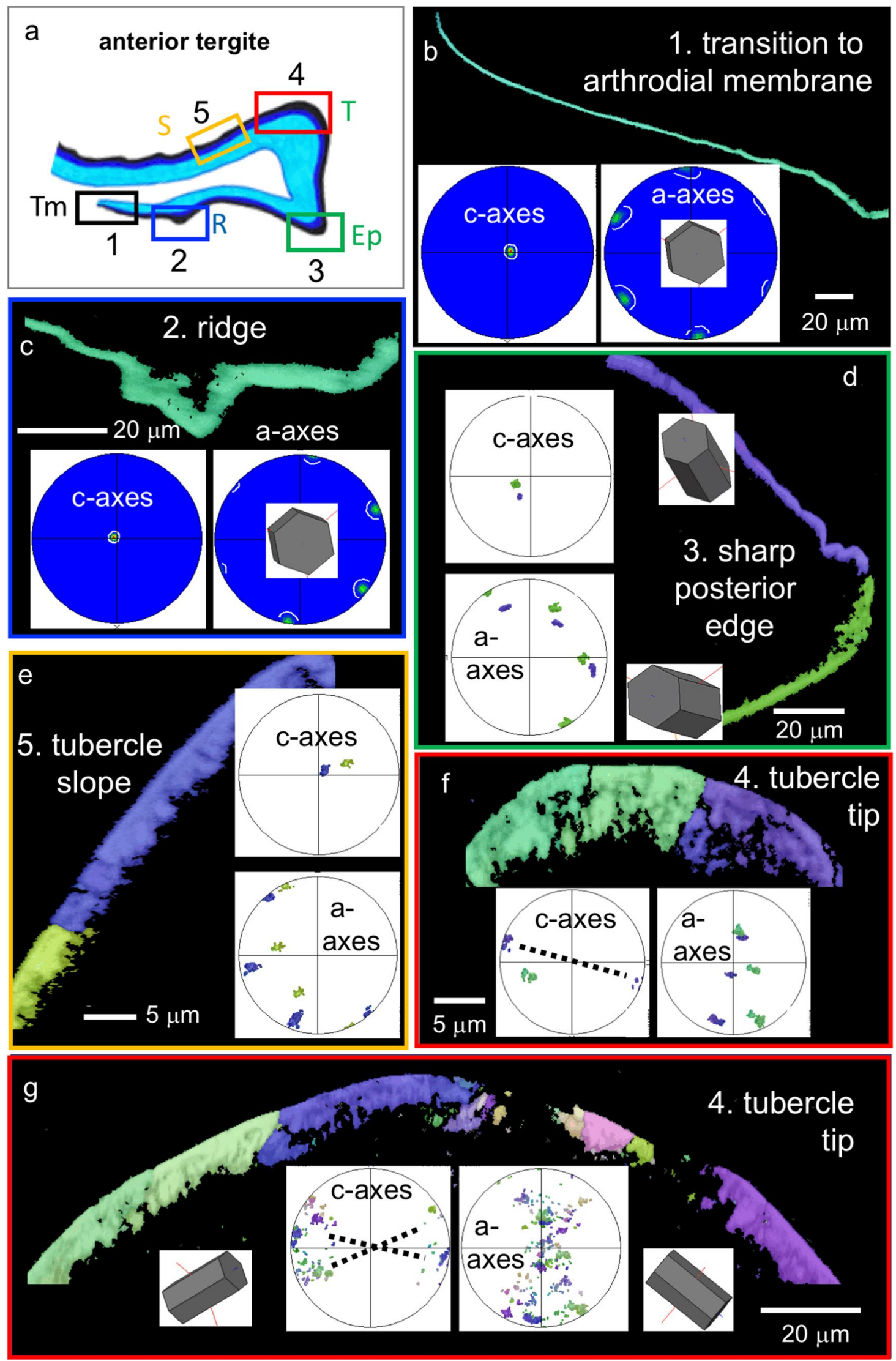
4Fig. 8 Microstructure and texture of the calcite layer at different regions of the ventral cuticle: a Schematic overview of the probed areas; coloured rectangle indicate skeletal portions that were scanned with EBSD, b Calcite organization between the ridge $(\mathrm{R})$ and the transition to arthrodial membrane (Tm), c Calcite orientation at the ridge, $\mathbf{d}$ Calcite assembly at the sharp posterior edge (Ep), e Calcite orientation at the tubercle slope $(\mathrm{S}),(\mathbf{f}, \mathbf{g})$ calcite organization at the tubercle tip (T). Two texture patterns are developed: a bimodal orientation distribution at the tip of the tubercle (f, $\mathbf{g})$ and an almost single-crystalline pattern of calcite orientation at the transition to the arthrodial membrane and at the ridge. C-axes orientations turn between the tubercle tip and the transition to the arthrodial membrane. In the former, calcite c-axes are within the plane of view, while in the latter c-axes orientations are perpendicular to the plane of view

muscle attachment [29], while in $H$. reaumuri the cuticle just forms a thicker bulge than in the median plain. This difference is likely due to the behaviour of T. europaeus upon predation. The animal can roll itself into a sphere to protect the soft ventral part of the body. This requires stronger muscles to maintain the spherical posture when a predator tries to unroll the animal explaining the larger attachment sites for the lateral muscles. Muscle strands attached to bulges have also been observed near the transition to the arthrodial membrane in the joint head between the pereiopods and the coxal plates of $P$. scaber, where they serve to stabilize the shape of smooth gliding faces avoiding friction with the joint socket [5]. In regions without muscle attachment, the bulge near the transition to the arthrodial membrane observed in $H$. reaumuri and $T$. europaeus likely serves to stabilize the edge mechanically.

For the ventral cuticle at the posterior edge of the tergites, we find both similarities and also large differences between T. europaeus and $H$. reaumuri (Fig. 11). The thickness of the ventral cuticle needs to taper off towards the thin arthrodial membrane, so that the ventral edge region gradually becomes more pliable. Like in T. europaeus, the cuticle of the posterior edge in $H$. reaumuri bends down and backwards to the anterior side hiding the thinner ventral side of the cuticle underneath the protective dorsal cuticle. In addition, the bend brings the arthrodial membrane deep between adjacent tergites, making it less accessible to the mouthparts of predators like the chelicera of spiders (Fig. 11). In T. europaeus, the ventral tergite cuticle is rather short, covering about one tenth of the dorsal cuticle length, whereas in $H$. reaumuri it covers about one-fifth. Thus, the distance between the sharp posterior edge and the beginning of the arthrodial membrane is twice as large in relation to the tergite length as that in T. europaeus, indicating a better protection of the arthrodial membrane against predators. In contrast, being a roller, the anterior part of the cuticle of $T$. europaeus that can slide underneath the posterior part of the preceding edge is with more than one-third of the tergite length much larger than in $H$. reaumuri, in which only one-fourth of the tergite's anterior part sits underneath the preceding one (Fig. 11a, b). In the cuticle of the edge region of the tergite of $H$. reaumuri we found two features that are not present in T. europaeus. These are the ridge region and the arched ventral cuticle region that spans from the ridge to the sharp posterior edge of the tergite. The similarity in the length and curvature of the arched ventral cuticle and the anterior part of the tergite cuticle suggests that these two parts fit well upon one another forming an arched thin gap between the two cuticle parts (Fig. 11b). Such an elongated arched gap can hinder a predator to reach the arthrodial membrane. Because the arthrodial membrane is likely more permeable for water vapor than the thick and hard skeletal elements the thin gap between tergites may furthermore function as a barrier for water loss. The ridge situated near the transition to the arthrodial membrane likely serves to stabilize the cuticle before it becomes radically reduced in thickness. The row of regularly spaced cone-shaped bristles at the ridges may serve to prevent dirt and sand grains to reach the arthrodial membrane. This is likely an adaptation to the burrowing behaviour of $H$. reaumuri. In the sand burrowing beach isopod T. europaeus rectangular wedge-shaped scales and elongated scales with long bristles at the posterior bend of the tergites serve a similar function, preventing sand grains to enter the cleft between the tergites when the animal unrolls from its spherical posture. In the anterior tergite the distance between the ridge and the arthrodial membrane is longer and thus the ventral arched cuticle shorter than in the posterior tergite, resulting in a less flexible arched ventral cuticle. The reason for this could be the functional differentiation of the anterior tergites. The tubercles on the anterior tergites increase their overall stiffness and bending strength according to the same principle as corrugated steel [30]. This plays a role in the digging behaviour of the animal besides the function of the tubercles in guarding the entrance to the burrow [20, 21, 31]. During burrowing, the animal removes soil particles first with its mouthparts. When it carries the particles out of the burrow with its first two pairs of walking legs, it crawls backwards and presses the tubercles of the anterior tergites against the burrow wall, removing additional soil grains. During this backwards movement, it is more likely that soil grains enter the space between neighbouring anterior tergites than between neighbouring posterior tergites. Here, a shorter distance between the ridge and the sharp posterior edge may be of advantage. The shorter distance result in a lower leverage the grains can exert to the ventral arched cuticle. Thus, the cone shaped bristles of the ridge are kept closer to the underlapping tergite preventing the grains to reach the arthrodial membrane. 

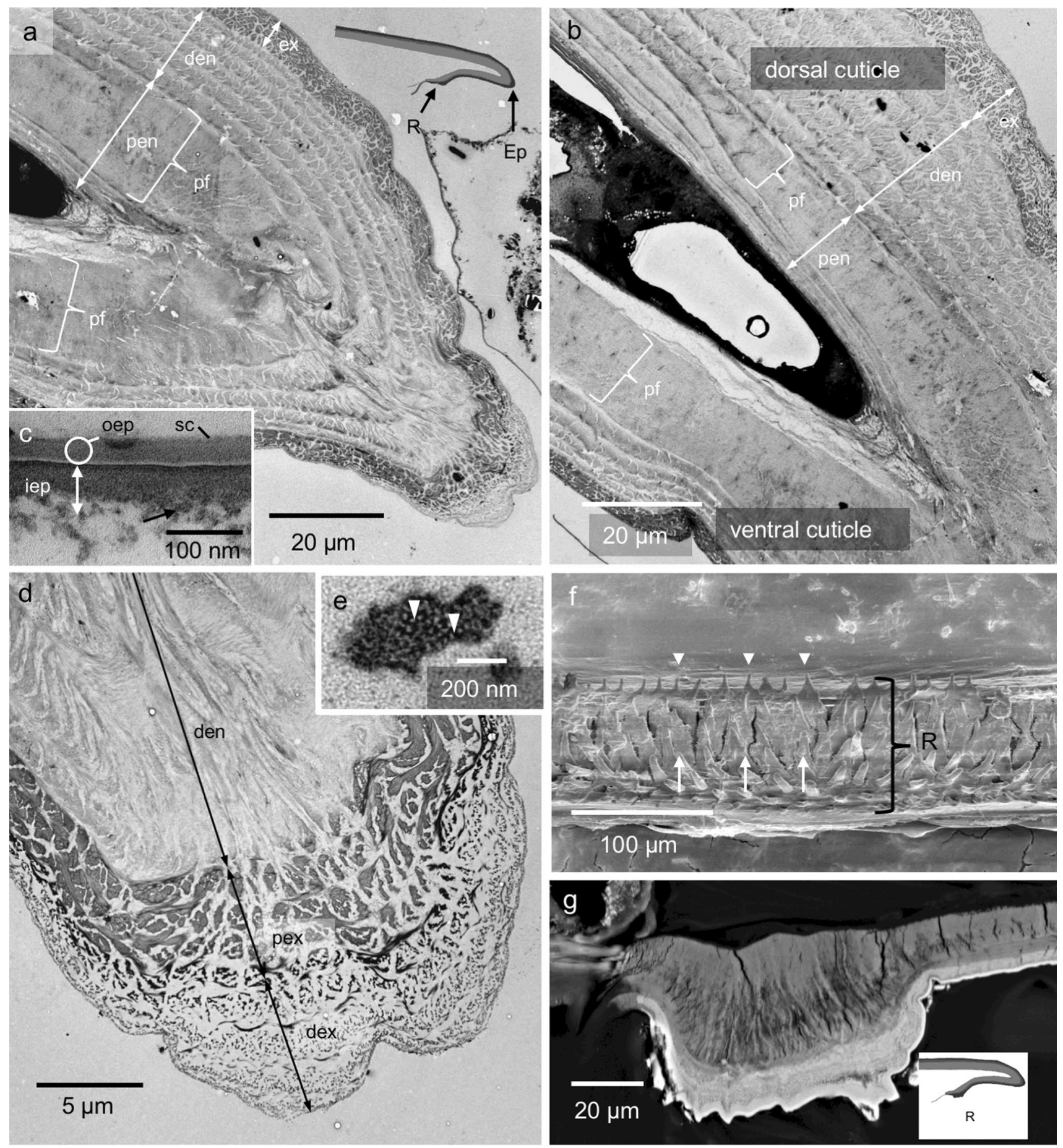

Fig. 9 Structure of the posterior edge and ventral region of a posterior tergite in Hemilepistus reaumuri. a-e TEM micrographs of sagittally cut samples. a, b Overviews of two adjacent regions in the sharp edge showing the exocuticle (ex), the distal (den) and proximal endocuticle (pen) and the layer of parallel oriented fibrils (pf). c The epicuticle at the edge; iep, inner epicuticle; oep, outer epicuticle; sc, surface coat. d Detail of the sharp posterior edge showing the changes in thickness of dex and pex. e Detail from the distal exocuticle showing a fibre consisting of chitin fibrils within a proteinaceous matrix. f SEM micrograph from the ventral side of the posterior tergite depicting the ridge $(\mathrm{R})$ of ventral cuticle. The arrowheads mark regular arranged cone shaped bristles and the arrows large scales. $\mathbf{g}$ BSE-SEM micrograph of the ridge. Dense regions appear brighter 


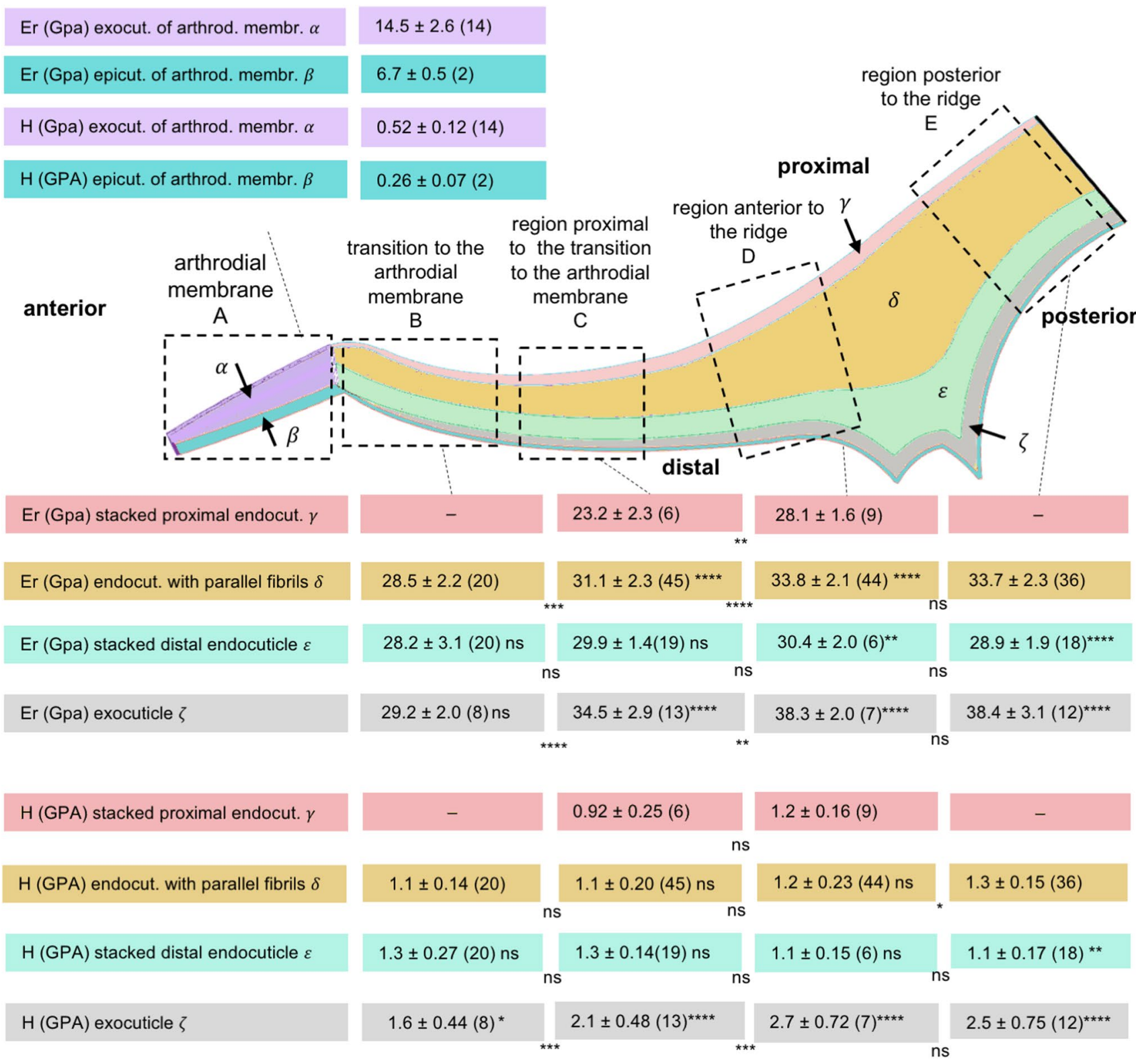

Fig. 10 Schematic depiction of the cuticle regions from the ridge to the arthrodial membrane tested with nanoindentation and the results providing mean values and standard deviations for the reduced elastic modulus (Er) and the hardness $(\mathrm{H})$ of various layers of the cuticle. The number of indents performed on each region is given in parentheses. Colours and of the boxes match the colours of the probed cuticle layers. Statistically significant differences were determined

\subsection{Ultrastructure of the edges and the ventral cuticle}

The principal layers of the edge and ventral cuticle are the same as in the dorsal tergites of $H$. reaumuri that has been described previously [18, 27]. However, the fusion of stacks to one large stack with fibrils oriented parallel to each other found in the anterior and posterior edge using two-way ANOVA. Holm-Sidiak multiple comparisons tests were used to determine significant differences of the regions. Asterisks on the right side of the mean values mark the significant differences between the mean values with that of the proximal neighbour and asterisks between boxes mark significant differences with the mean value of the neighbour at the anterior side. ${ }^{*} p<0.5$, $* * p<0.01$, *** $p<0.001, * * * * p<0.0001$, ns: not significant

and the ventral cuticle is unusual. The orientation of the fibrils largely affects the mechanical properties of the cuticle because of the high anisotropy of the elastic modulus of chitin, which is $119 \mathrm{GPa}$ in the axial direction and 29 GPa perpendicular to it $[32,33]$. This explains why the elastic modulus within the region of the endocuticle, in which unidirectional oriented fibrils point to the lateral sides of the tergite, is higher than in the distal endocuticle 


\section{a Tylos europaeus}

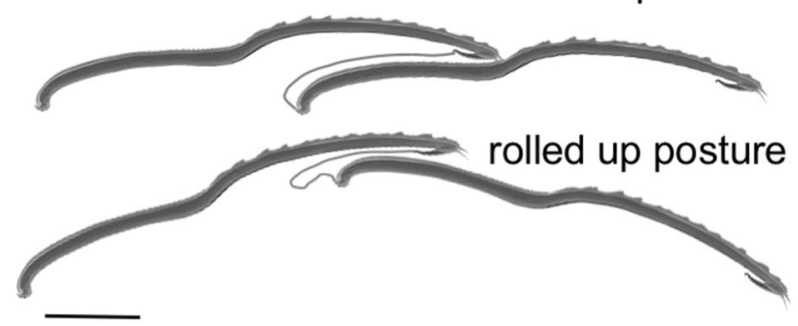

$0.5 \mathrm{~mm}$

b

Hemilepistus reaumuri

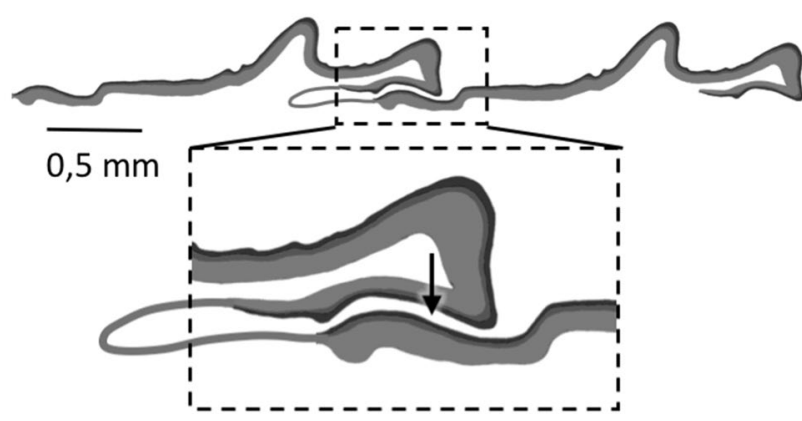

Fig. 11 Schemes of the shape and spatial relation of two neighbouring tergites. a the relative position of tergites in Tylos europaeus in its rolled up und unrolled position. b The tergites of Hemilepistus reaumurii form a thin gap (arrow) between the ventral cuticle of the tergite and the underlapping anterior region of the next posterior tergite

containing rotating stacks of fibrils. Within the bulge of the anterior edge the orientation of the fibril's long axis to the lateral sides increases the bending strength of the cuticle. This is a similar situation as in the two principal veins running along the anterior edge of the wings of a termite. In these the chitin fibrils orientation run parallel to the veins, maximizing the bending strength perpendicular to their longitudinal direction [34]. Another interesting example is the highly stretchable intersegmental membranes in the abdomen of the female migratory locust. These allow the abdomen to be stretched out to dig a deep hole for egg deposition. In the intersegmental membrane, the chitin fibrils are oriented normal to the direction of abdominal expansion, so that the membrane can be stretched more than 12 times their original length [35]. At the posterior side of the tergite, were the stack of parallel fibrils runs from the dorsal edge region to the ventral cuticle and all the way to the arthrodial membrane, the orientation of fibrils may affect the mechanical properties in two distinct manners. At the posterior edge and the ridge, which are short and thick in comparison to the ventral arched cuticle region and the region anterior to the ridge, the parallel fibrils increase the bending strength similar to those in the anterior bulge region. In long and thin cuticle regions of the arched ventral cuticle and the cuticle between the ridge and the transition to the arthrodial membrane; however, the fibril orientation towards the lateral sides mainly facilitates flexion of the cuticle between dorsal and ventral positions. Fused stacks with unidirectional fibril orientation have not been found in the edge regions of tergites in $T$. europaeus, but in two different skeletal elements of $P$. scaber. Within a large part of the endocuticle, in the partes incisivae of the mandibles, parallel fibrils are oriented in the direction to the cutting edge, resulting in higher local stiffness and hardness in this direction [9]. In the beforementioned bulge at the transition to the arthrodial membrane within the joint head of $P$. scaber the fibrils run in parallel within the whole endocuticle. Here the fibrils are oriented in the direction of the force exerted by muscles that attach to the bulge [5].

\subsection{Ultrastructure at the transition to the arthrodial membrane}

The increase in the thickness of the epicuticle just at the transition to the arthrodial membrane, the concomitant disappearance of the distal exocuticle, the increase in the phosphorus content and reduction in mineral content has been observed previously in the edge regions of the tergite in $T$. europaeus [19] and in the joint head of $P$. scaber [5]. Therefore, these features are likely common for cuticular edge regions in isopods. The epicuticle is thought to help preventing excessive water loss by evaporation [28]. Since the arthrodial membrane is much thinner than the cuticle of the sclerites, one might expect that in the arthrodial membrane of the desert isopod the epicuticle should be in particular thick. This, however, is not the case. In the joint head of $P$. scaber that lives in mesic habitats, and T. europaeus that has ready access to seawater, the epicuticle of the arthrodial membrane is, with about $1 \mu \mathrm{m}$, of similar thickness than in $H$. reaumuri. This emphasizes the above-mentioned role of the long ventral part of the tergite cuticle to reduce water loss through evaporation along the gap between the arthrodial membrane and the cuticle.

There is some variation in the layers of the exo- and endocuticle that continue into the arthrodial membrane. In $H$. reaumuri, T. europaeus and Callinectes sapidus both, the proximal exocuticle and stacks of the endocuticle enter the arthrodial membrane, whereas in the joint head of $P$. scaber only the proximal exocuticle continues into the arthrodial membrane, whereas the endocuticle, which consists of a stack of parallel fibrils, does not. [5, 19, 36]. Interestingly, in the aforementioned inter-segmental membrane of the migratory locust, the endocuticle and not the exocuticle continues 
into the membrane [35]. It has been suggested that the difference between T. europaeus and P. scaber is species specific, because in T. europaeus the exocuticle consist almost exclusively of the distal exocuticle and the proximal exocuticle is very thin.

\subsection{Mineral phases: their distribution, composition and calcite orientation}

Our previous study on the dorsal cuticle of the tergites of $H$. reaumuri has shown that the main mineral phases within the exo- and endocuticle of $H$. reaumuri are $\mathrm{Mg}$-calcite, ACC and minor amounts of ACP [18]. Elemental maps and EBSD measurements indicate that these mineral phases are present in the posterior edge and in the ventral cuticle. The distribution of calcite within the posterior edge and ventral cuticle, as measured with EBSD, is about congruent with the location of the exocuticle. The mineral phase within the endocuticle should therefore be ACC, as we did not obtain a backscatter diffraction signal. This distribution pattern of mineral phases has been found in the tergite cuticle of $P$. scaber and $A$. vulgare $[3,7]$ which, like $H$. reaumuri, belong to the Crinochaeta, which is the largest taxon of terrestrial isopods. In T. europaeus, a member of the Tylidae, calcite is restricted to the distal exocuticle, and ACC to the very thin proximal exocuticle and the endocuticle $[4,19]$. In the dorsal cuticle of $H$. reaumuri, calcite forms as a dense layer within the exocuticle. In addition, calcite has been found within a distal region of the endocuticle as well [18]. EBSD maps have shown that in the dorsal endocuticle calcite appears as sparsely dispersed grains [18]. Interestingly, not all Kikuchi patterns recorded from this region could automatically be indexed by the EBSD acquisition software during measurement. This indicates the presence of calcite crystallites that, most probably due to their minute size, could not be distinguished from ACC domains [18]. The distribution pattern of calcite at the sharp posterior edge and in the ventral cuticle differs significantly from that in the dorsal cuticle, tubercle tips and non-tubercular regions of the anterior cuticle (Figs. 8, S1 and [18]). The sharp posterior edge and the ventral cuticle lacks dispersed calcite in the endocuticle, only the distal dense calcite layer is present (Figs. S1b, c). From the dorsal cuticle to the sharp posterior edge and the back folded, ventral cuticle of the anterior tergite mineral unit sizes and crystal co-orientation strengths increase (Fig. 12c, d). The calcite layer of the back folded section consists of two mineral units: (i) one that extends from the sharp posterior edge and covers the arced ventral cuticle and (ii) one mineral unit comprising the calcite layer at the ridge and the cuticle towards and near the transition to the arthrodial membrane. Calcite in both mineral units is highly co-oriented, almost single crystalline. Calcite crystal orientation changes between tubercle tip, the sharp posterior edge and the transition to the arthrodial membrane (Fig. 8). At the tip, it is either within the plane of view of the sagittal cut sample or only slightly tilted out of it. Calcite crystals become more tilted out of the plane of view at the sharp posterior edge and are $90^{\circ}$ tilted out of the plane of view at the transition to the arthrodial membrane (Figs. 8, 12). This strict change in crystal orientation is highly remarkable and might be a feature that is intrinsic to terrestrial isopods, because it has also been observed for T. europaeus (Fig. 12e and [19]. For both isopod species, the bend at the sharp posterior edge is the starting point for the change in calcite crystal orientation between dorsal cuticle portions, the back folded ventral cuticle and, especially, the calcite at the very end of the calcite layer at the transition to the arthrodial membrane.

Besides the orientation of fibrils within the cuticle, the amount of mineral, the mineral composition and the type of the mineral phase dominate the local mechanical properties of the cuticle. The difference of the values for $\mathrm{Er}$ and $\mathrm{H}$ between the proximal and distal stacks of the ventral endocuticle, both with a twisted fibril orientation (in particular in the region C of Fig. 10), is due to the lower mineral content in the proximal stacks, as indicated by the elemental maps. Cuticle mineralized with calcite is stiffer and harder than cuticle mineralized with ACC [1]. This explains the up to $25 \%$ and $60 \%$ higher values for $\mathrm{Er}$ and $\mathrm{H}$, respectively, within the exocuticle in comparison to the stacked distal endocuticle. However, variations in mineral phase and content may not be the only factors that lead to gradients in stiffness and hardness in the cuticle. It has been shown that the local elastic modulus can be adapted to function by varying the amount of the elastic protein resilin, and the degree of cuticle sclerotization. In insects those variations are used to produce gradients in the elastic modulus of the cuticle at the micrometer scale [37]. Such gradients can provide an optimal compromise between load bearing capacity and resilience [38]. Comparison of the reduced elastic modulus and hardness of the ventral cuticle presented here with that of the dorsal tergite cuticle [18] gave similar values for the stacked endocuticle with twisted fibril orientation and almost 20\% higher values in the calcite containing layers of dorsal cuticle. This difference is likely due to a high mineral content of the dorsal cuticle.

It is of interest that the sharp posterior edge and the ridge are strengthened with the thickest calcite layer of 6 to $8 \mu \mathrm{m}$ and by an increase in cuticle thickness. The posterior sharp edge can be exposed to high external mechanical loads, e.g. if a predator tries to widen the gap between two neighbouring tergites. Strengthening of the edge may help to protect the thinner ventral cuticle parts. At the posterior edge of $T$. europaeus neither the cuticle thickness nor the thickness of the calcite layer is changed [19]. At the ridge of $H$. reaumuri, the increase in the overall cuticle thickness and the thickness of the calcite layer increase the stability against bending in 


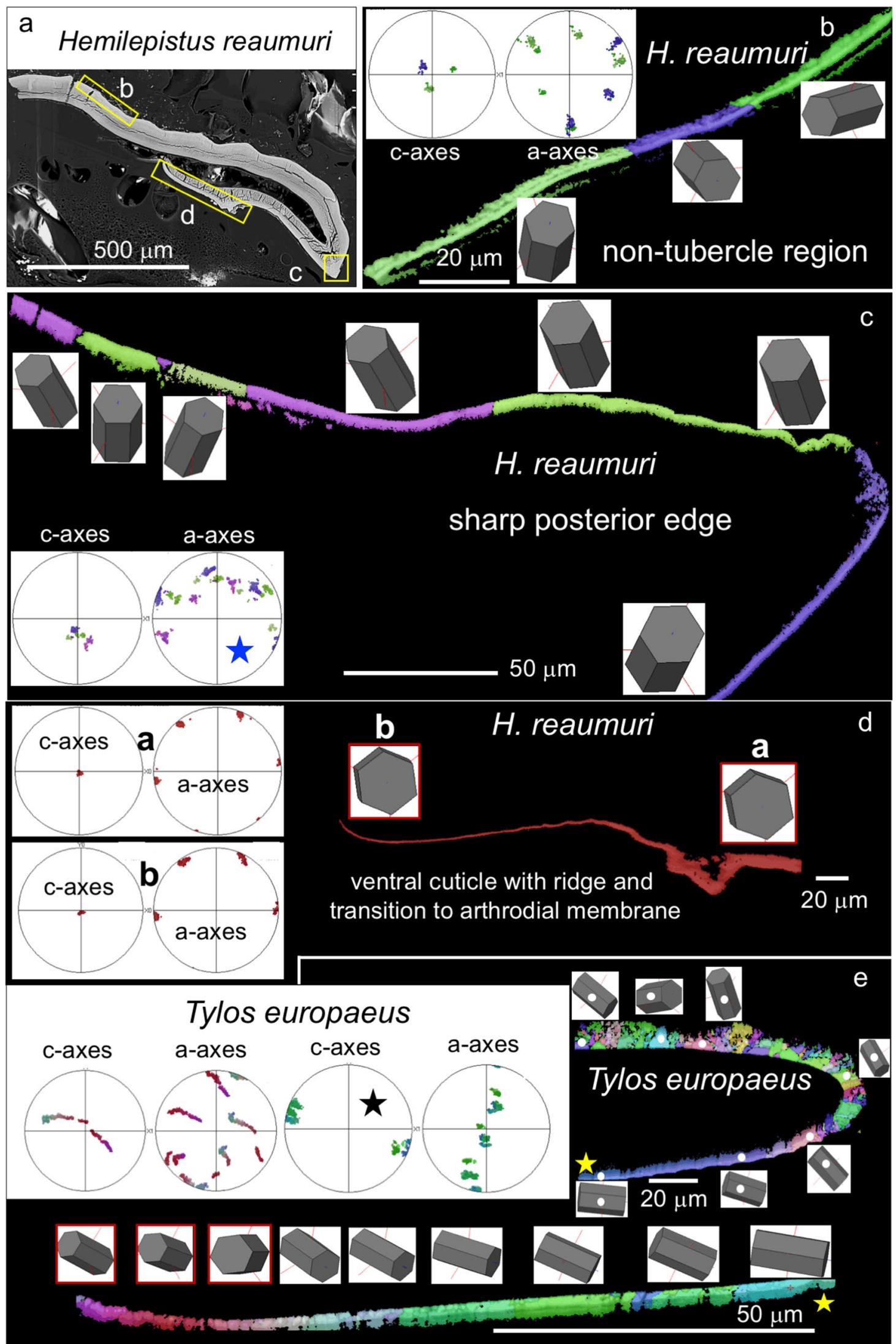


4Fig. 12 Difference in calcite organization within the calcite layer in ventral cuticle portions between Hemilepistus reamuri (a-d) and Tylos europaeus (e). Calcite crystal organization in T. europaeus changes from almost random at tubercular tergite portions to graded in the ventral cuticle; gradation starts at the sharp posterior edge (e and [19]). In $H$. reamuri calcite crystallites are weakly co-oriented at non-tubercular regions (b), increase in co-orientation strength close to the sharp posterior edge (c) and are almost single crystalline within the ventral cuticle: at the ridge and at the transition to the arthrodial membrane (d). For both, $H$. reamuri and $T$. europaeus calcite c-axes at the very tip of the transition to the arthrodial membrane point $90^{\circ}$ out of the plane of view (d, e), while for T europaeus at ventral cuticle portions calcite c-axes are within (black star in the pole figure in e) and for ventral and dorsal cuticle of $H$. reamuri are tilted out of the plane of view by 60-70 degrees (blue star in the pole figure in c)

addition to the layer of parallel fibrils pointing to the lateral sides. The decrease in thickness of the cuticle and its calcite layer by a factor of about 2 from the anterior side of the ridge to the transition of the arthrodial membrane successively increases the flexibility of the cuticle. The accompanied successive decrease in the reduced elastic modulus and hardness in the exocuticle and of the elastic modulus within the layer of fibrils oriented in parallel further increase the flexibility of the cuticle.

The phosphorus content within the anterior and posterior edges and ventral cuticle regions of both the anterior and posterior tergites indicates the presence of ACP. Small amounts of ACP are common in Crustacea [1, 39-41] including Isopoda $[1,11]$. In most regions, the ratios between phosphorus and calcium are similar to those observed within the dorsal tergite cuticle of $H$. reaumuri [18]. However, at the transition to the arthrodial membrane the phosphorus content is exceptionally high, indicating a high contribution of ACP to the cuticle mineral. It has been shown that ACP instead of ACC decreases both the local hardness as well as the stiffness of the cuticle $[9,42$, 43]. This is in accordance with the lower values measured for the local mechanical properties at the transitions to the arthrodial membranes found in the present study and those reported for T. europaeus [19]. Even higher calcium/phosphorus ratios exist within the arthrodial membranes of $H$. reaumuri that contain mineral at their margins. Mineral was absent in the arthrodial membranes of T. europaeus and $P$. scaber, but has been previously described in those of decapod Crustacea [1].

\section{Summary and conclusions}

The tergites are the main load bearing skeletal elements of isopods. They are connected by flexible arthrodial membranes to allow for relative movements of the tergites. The desert ispod H. reaumuri is of particular interest because of its extreme habitat and the related social behaviour. Comparisons with the beach isopod T. europaeus indicate that in particular the posterior tergite edge regions are subject to evolutionary adaptations to the animal's habitat and behaviour.

1. The anterior edges at the tergites appear rather simple. They form a bulge that stabilizes the transition to the arthrodial membrane and laterally attachment sites for muscles. This appears to be a common feature in terrestrial isopods. The presence of large apodemes for muscle attachment in $T$. europaeus is likely due to its rolling behaviour to avoid predation.

2. The more complex morphology of the ventral cuticle in $H$. reaumuri as compared to $T$. europaeus indicates a functionalization related to the extreme desert habitat. A thin arched gap that can be formed by the anterior part of the tergite and a long ventral cuticle of the posterior edge region may (i) reduce water loss through evaporation from the arthrodial membrane and (ii) protect the arthrodial membrane from the mouthparts of predators. A thick ridge stabilizes the otherwise thin ventral cuticle. Cone-shaped bristles on the ridge prevent sand grains from access to the arthrodial membrane.

3. An unusual large stack with parallel fibrils oriented to the lateral sides, found in the anterior and posterior edge region of $H$. reaumuri tergites are lacking in T. europaeus. They increase the bending strength of the cuticle in the direction perpendicular to the fibrils long axes. In the posterior edge region, they likely serve to facilitate flexion of the cuticle in the dorsal and ventral direction. The decrease in thickness of the cuticle and its calcite layer, the local stiffness and hardness successively further increase the flexibility of the cuticle.

4. An increase in the thickness of the epicuticle to about $1 \mu \mathrm{m}$ at the transition to the arthrodial membrane, a concomitant disappearance of the calcite layer, an increase in ACP content and a reduction in calcium carbonate content appear to be common characteristics for the transitions of terrestrial isopods. Likely they improve the pliability of the cuticle to prevent cuticle failure during frequently repeating mechanical loading caused by relative movements of neighbouring tergites.

5. The calcite layer within anterior tergite cuticle varies significantly in thickness, specificity and orientation of constituting calcite crystallites. At the dorsal tergite cuticle the calcite layer consists of a dense distal and a patchy proximal part. The sharp posterior edge, and the ventral cuticle is reinforced only by the distally located dense calcite layer. Calcite mineral unit size and calcite co-orientation strength increase towards the sharp posterior edge and, especially, towards the transition to the arthrodial membrane. An about $90^{\circ}$ rotation of calcite 
c-axes is present between the tubercle tip and the very end of the calcite layer next to the arthrodial membrane. Calcite crystallites adjacent to the arthrodial membrane are tilted about $90^{\circ}$ out of the plane of view, a feature that is also observed for the back folded ventral cuticle in T. europaeus.

Acknowledgements We are grateful to Heidi Bögershausen, MaxPlanck-Institut für Eisenforschung $\mathrm{GmbH}$, Düsseldorf, for help with the nanoindentation experiments.

Funding Open Access funding provided by Projekt DEAL. This work was supported by the Deutsche Forschungsgemeinschaft (DFG) within the priority programs SPP 1420 (Zi 368/8-1.

Availability of data and materials The datasets generated during and/ or analyzed during the current study are not publicly available but are available from the corresponding author on reasonable request.

\section{Compliances with ethical standards}

Conflict of interest The authors declare that they have no conflict of interest.

Ethics approval The study complies with the ethical standards on the welfare of animals of the European Union.

Open Access This article is licensed under a Creative Commons Attribution 4.0 International License, which permits use, sharing, adaptation, distribution and reproduction in any medium or format, as long as you give appropriate credit to the original author(s) and the source, provide a link to the Creative Commons licence, and indicate if changes were made. The images or other third party material in this article are included in the article's Creative Commons licence, unless indicated otherwise in a credit line to the material. If material is not included in the article's Creative Commons licence and your intended use is not permitted by statutory regulation or exceeds the permitted use, you will need to obtain permission directly from the copyright holder. To view a copy of this licence, visit http://creativecommons.org/licenses/by/4.0/.

\section{References}

1. H.-O. Fabritius et al., Bioinspiration. Biomimetics 11, 055006 (2016)

2. S. Hild, F. Neues, N. Žnidaršič, J. Štrus, M. Epple, O. Marti, A. Ziegler, J. Struct. Biol. 168, 426 (2009)

3. S. Hild, O. Marti, A. Ziegler, J. Struct. Biol. 163, 100 (2008)

4. B. Seidl, K. Huemer, F. Neues, S. Hild, M. Epple, A. Ziegler, J. Struct. Biol. 174, 512 (2011)

5. S. Ruangchai, C. Reisecker, S. Hild, A. Ziegler, J. Struct. Biol. 182, 22 (2013)

6. B.H.M. Seidl, A. Ziegler, ZooKeys 176, 73 (2012)

7. B.H.M. Seidl, C. Reisecker, S. Hild, E. Griesshaber, A. Ziegler, Z. Kristallogr. 227, 777 (2012)

8. J. Huber, E. Griesshaber, F. Nindiyasari, W.W. Schmahl, A. Ziegler, J. Struct. Biol. 190, 173 (2015)

9. J. Huber, H.-O. Fabritius, E. Griesshaber, A. Ziegler, J. Struct. Biol. 188, 1 (2014)

10. A. Becker, U. Bismayer, M. Epple, H. Fabritius, B. Hasse, J. Shi, A. Ziegler, Dalton. Trans. 2003, 551 (2003)
11. F. Neues, A. Ziegler, M. Epple, Crystal. Eng. Com. 9, 1245 (2007)

12. K. Pütz, F. Buchholz, Mar. Biol. 110, 49 (1991)

13. A.C. Neville, D.A.D. Parry, J. Woodhead-Galloway, J. C. Sci. 21, 73 (1976)

14. Y. Bouligand, Tissue. Cell. 4, 189 (1972)

15. V.B. Wigglesworth, Tissue. Cell. 17, 227 (1985)

16. N.F. Hadley, M.R. Warburg, Comp. Biochem. Physiol. 85A, 669 (1986)

17. A. Becker, A. Ziegler, M. Epple, Dalton. Trans. 2005, 1814 (2005)

18. F. Ernst, H.-O. Fabritius, E. Griesshaber, C. Reisecker, F. Neues, M. Epple, W.W. Schmahl, S. Hild, A. Ziegler, J. Struct. Biol. 212, $107570(2020)$

19. B.H.M. Seidl, E. Griesshaber, H.-O. Fabritius, C. Reisecker, S Hild, S. Taiti, W.W. Schmahl, A. Ziegler, J. Struct. Biol. 204, 464 (2018)

20. H. Schmalfuss, Symp. Zool. Soc. Lond. 53, 49 (1984)

21. K. E. Linsenmair, Verhandlungen der Deutschen Zoologischen Gesellschaft, 60 (1979)

22. E.K. Linsenmair, C. Linsenmair, Z. Tierpsychol. 29, 134 (1971)

23. H. Fabritius, P. Walther, A. Ziegler, J. Struct. Biol. 150, 190 (2005)

24. E. Griesshaber, R.D. Neuser, W.W. Schmahl, Seminarios SEM. 7, $22(2010)$

25. M. Greiner, X. Yin, L. Fernández-Díaz, E. Griesshaber, F. Weitzel, A. Ziegler, S. Veintemillas-Verdaguer, W.W. Schmahl, Cryst. Growth. Des. 18, 1401 (2018)

26. W.C. Oliver, G.M. Pharr, J. Mater. Res. Soc. 7, 1564 (1992)

27. A. Ayari, M. Raimond, C. Souty-Grosset, K. Nasri-Ammar, J. Struct. Biol. 193, 115 (2016)

28. P. Compère, in The Biology of Terrestrial Isopods III, edited by P. Juchault, and J. P. Mocquard (Université de Portiers, 1990), pp. 169

29. F. Erhard, Stuttgarter. Beiträge. zur. Naturkunde. 550, 1 (1997)

30. H. Schmalfuss, Stuttgarter Beiträge zur Naturkunde 17, 1 (1983)

31. H. Schmalfuss, Zeitschrift für Morphologie der Tiere 80, 287 (1975)

32. S. Nikolov, M. Petrov, L. Lymperakis, M. Friák, C. Sachs, H.-O Fabritius, D. Raabe, J. Neugebauer, Adv. Mater. 22, 519 (2010)

33. J.F.V. Vincent, U.G.K. Wegst, Arthopod. Struc. Dev. 33, 187 (2004)

34. R. Hulme, Nature 175, 128 (1955)

35. J.F.V. Vincent, Tissue. Cell. 13, 831 (1981)

36. E.E. Williams, M.J. Anderson, T.J. Miller, S.D. Smith, Comp. Biochem. Physiol. B 137, 235 (2004)

37. S. Eshghi, M. Jafarpour, A. Darvizeh, S.N. Gorb, H. Rajabi, J.R. Soc, Interface 15, 20180312 (2018)

38. M. Jafarpour, S. Eshghi, A. Darvizeh, S.N. Gorb, H. Rajabi, J.R. Soc, Interface 17, 20200378 (2020)

39. P. Greenaway, Biol. Rev. 60, 425 (1985)

40. R. Roer, R. Dillaman, Am. Zool. 24, 893 (1984)

41. K.K. Vijayan, A.D. Diwan, Comp. Biochem. Physiol. 114A, 91 (1996)

42. H.-O. Fabritius, C. Sachs, P.R. Triguero, D. Raabe, Adv. Mater. 21, 391 (2009)

43. Y. Politi, B. Bar-On, and H.-O. Fabritius, in Architectured Materials in Nature and Engineering, edited by Y. Estrin, R., Bréchet, Y., Dunlop, J. and Fratzl, P. (Springer Series in Materials Science, Cham, 2019), pp. 287

Publisher's Note Springer Nature remains neutral with regard to jurisdictional claims in published maps and institutional affiliations. 